\title{
On Feller and Strong Feller Properties and Irreducibility of Regime-Switching Jump Diffusion Processes with Countable Regimes*
}

\author{
Khwanchai Kunwai and Chao Zhu \\ Department of Mathematical Sciences, University of Wisconsin-Milwaukee, Milwaukee, WI \\ 53201, USA, kkunwai@uwm.edu, zhu@uwm. edu
}

August 18, 2020

\begin{abstract}
This work focuses on a class of regime-switching jump diffusion processes with a countably infinite state space for the discrete component. Such processes can be used to model complex hybrid systems in which both structural changes, small fluctuations as well as big spikes coexist and are intertwined. The paper provides weak sufficient conditions for Feller and strong Feller properties and irreducibility for such processes. The conditions are presented in terms of the coefficients of the associated stochastic differential equations.
\end{abstract}

Keywords: Regime-witching jump diffusion, Feller property, strong Feller property, irreducibility.

Mathematics Subject Classification: 60J27, 60J60, 60J75, 60G51

\section{Introduction}

Motivated by the increasing need of modeling complex systems, in which both structural changes and small fluctuations as well as big spikes coexist and are intertwined, this paper continues the study on regime-switching jump diffusion processes with countable regimes. Our focus is on Feller and strong Feller properties and irreducibility for such processes. We provide weak sufficient conditions for Feller and strong Feller properties and irreducibility.

Roughly speaking, a regime-switching jump diffusion process can be considered as a two component process $(X(t), \Lambda(t))$, an analog (or continuous state) component $X(t)$ and a switching (or discrete event) component $\Lambda(t)$. The analog component models the state of interest while the switching component can be used to describe the structural changes of the state or random environment or random factors that are not represented by the usual jump diffusion formulation. For instance, a regime-switching Black-Scholes model is considered in Zhang (2001), in which the continuous component $X(t)$ models the price evolution of a risky asset and the switching component $\Lambda(t)$ delineates the overall economy state. Regime-switching jump diffusion is also used in

${ }^{*}$ This research was supported in part by the Simons Foundation (grant award number 523736) and a DIG award from the University of Wisconsin-Milwaukee. The first author was also supported by the Development and Promotion of Science and Technology Talents project (DPST). 
mathematical biology such as the recent paper Tuong, Nguyen, Dieu \& Tran (2019), in which a stochastic SIRS model subject to both white and color noises is analyzed. We refer to Mao \& Yuan (2006), Shao (2015a,b), Shao \& Xi (2014), Yin \& Zhu (2010) and the references therein for more work on regime-switching jump diffusions and their applications.

In the theory of Markov processes and their applications, dealing with a Markov process $\xi(t)$ with $\xi(0)=x$, for a suitable function $f$, often one must consider the function $P_{t} f(x):=\mathbb{E}_{x}[f(\xi(t))]$. Following Dynkin (1965), the process $\xi(t)$ is said to be Feller if $P_{t} f$ is continuous for any $t \geq 0$ and $\lim _{t \downarrow 0} P_{t} f(x)=f(x)$ for any bounded and continuous function $f$ and it is said to be strong Feller if $P_{t} f$ is continuous for any $t>0$ and any bounded and measurable function $f$. This is a natural condition in physical or social modeling: a slight perturbation of the initial data should result in a small perturbation in the subsequent movement. In addition, Feller and strong Feller properties are intrinsically related to the existence and uniqueness of an invariant measure of the underlying process; see, for example, Meyn \& Tweedie (1992, 1993a,b).

While Feller and strong Feller properties for regime-switching (jump) diffusion processes have been investigated in the literature, this paper makes substantial improvements over the literature. It presents weak local non-Lipschitz conditions for Feller and strong Feller properties. A standing assumption in the literature (such as Nguyen, Yin \& Zhu (2017), Shao (2015b), Xi \& Zhu (2017), Yin \& Zhu (2010)) is that the coefficients of the associated stochastic differential equations are (locally) Lipschitz. While it is a convenient assumption, it is rather restrictive in many applications. For example, the diffusion coefficients in the Feller branching diffusion and the Cox-Ingersoll-Ross model are only Hölder continuous. For another example, many control and optimization problems often require the handling of systems where the (local) Lipschitz condition is violated. Motivated by these considerations, this paper further improves the results in the recent paper Xi, Yin \& Zhu (2019) by presenting weak non-Lipschitz conditions for Feller and strong Feller properties. The sufficient conditions are spelled out in Theorems 1.4 and 1.7. While certain technical aspects of the analyses are similar in both papers, the assumptions on the coefficients of the associated stochastic differential equations in this paper are substantially weakened; see Remarks 1.5 and 1.8 for details. It is also worth mentioning that the sufficient condition for strong Feller property in Theorem 1.7 is inspired by Priola \& Wang (2006), which deals with gradient estimate for diffusion semigroups. The extension from diffusions to regime-switching jump diffusions with countable regimes is nontrivial as the interactions between the analog and switching components add much subtlety and difficulty to the analyses.

The paper next considers irreducibility of regime-switching jump diffusions. Irreducibility is a topological property of the underlying stochastic process. Roughly speaking, irreducibility says that every point in the state space is reachable from any other point in the state space; see Section 1.1 for the precise definition. Irreducibility plays an important role in establishing the uniqueness of an invariant measure for the underlying Markov process; see, for example, Cerrai (2001), Hairer (2016). Unfortunately such a property for regime-switching jump diffusions has not been systematically investigated in the literature yet. In this paper, we derive irreducibility for regime-switching jump diffusions (Theorem 1.12) by using an important identity concerning the transition probability of such processes. An intermediate step, which is interesting in its own right, is to show that the subsystems consists of jump diffusions are irreducible under weaker conditions than those in the recent papers such as Qiao (2014) and Xi \& Zhu (2019). As an application, we present in Proposition 4.6 a set of sufficient conditions under which a unique invariant measure for regime-switching jump diffusions exists.

The rest of the paper is arranged as follows. We give the precise formulation of regime-switching jump diffusion processes in Section 1.1. The main results of the paper are summarized in Section 
1.2. Feller and strong Feller properties for regime-switching jump diffusions are established in Sections 2 and 3, respectively. Section 4 derives irreducibility for regime-switching jump diffusions. Two examples are studied in Section 5 for demonstration. Appendix A contains several technical proofs.

\subsection{Formulation}

Let $(U, \mathfrak{U})$ be a measurable space, $\nu$ a $\sigma$-finite measure on $U$, and $\mathbb{S}=\{1,2, \ldots\}$. Assume further that $d \geq 1$ is an integer, $b: \mathbb{R}^{d} \times \mathbb{S} \rightarrow \mathbb{R}^{d}, \sigma: \mathbb{R}^{d} \times \mathbb{S} \rightarrow \mathbb{R}^{d \times d}$, and $c: \mathbb{R}^{d} \times \mathbb{S} \times U \rightarrow \mathbb{R}^{d}$ are Borel measurable functions. Suppose $(X, \Lambda)$ is a right continuous, strong Markov process with lefthand limits on $\mathbb{R}^{d} \times \mathbb{S}$ such that the first component $X$ satisfies the following stochastic differential equation $(\mathrm{SDE})$,

$$
d X(t)=b(X(t), \Lambda(t)) d t+\sigma(X(t), \Lambda(t)) d W(t)+\int_{U} c\left(X\left(t^{-}\right), \Lambda\left(t^{-}\right), u\right) \tilde{N}(d t, d u)
$$

where $W$ is a standard $d$-dimensional Brownian motion, $N$ is a Poisson random measure on $[0, \infty) \times$ $U$ with intensity $d t \nu(d u)$, and $\tilde{N}$ is the associated compensated Poisson random measure. Here the second component $\Lambda$ is supposed to be a continuous-time stochastic process taking values in the set $\mathbb{S}$ and satisfies

$$
\mathbb{P}\{\Lambda(t+\Delta)=l \mid \Lambda(t)=k, X(t)=x\}= \begin{cases}q_{k l}(x) \Delta+o(\Delta) & \text { if } k \neq l \\ 1+q_{k l}(x) \Delta+o(\Delta) & \text { if } k=l\end{cases}
$$

uniformly in $\mathbb{R}^{d}$, provided that $\Delta \downarrow 0$.

To obtain the structure of the process $\Lambda$, let us consider the family of disjoint intervals $\left\{\Delta_{k l}(x)\right.$ : $\left.k, l \in \mathbb{S}, x \in \mathbb{R}^{d}\right\}$ defined on the positive half of the real line as follows:

$$
\begin{aligned}
\Delta_{12}(x) & =\left[0, q_{12}(x)\right), \\
\Delta_{13}(x) & =\left[q_{12}(x), q_{12}(x)+q_{13}(x)\right), \\
& \vdots \\
\Delta_{21}(x) & =\left[q_{1}(x), q_{1}(x)+q_{21}(x)\right), \\
\Delta_{23}(x) & =\left[q_{1}(x)+q_{21}(x), q_{1}(x)+q_{21}(x)+q_{23}(x)\right), \\
& \vdots \\
\Delta_{31}(x) & =\left[q_{1}(x)+q_{2}(x), q_{1}(x)+q_{2}(x)+q_{31}(x)\right),
\end{aligned}
$$

where $q_{k}(x):=\sum_{l \in \mathbb{S} \backslash\{k\}} q_{k l}(x)$ and we set $\Delta_{k l}(x):=\emptyset$ if $q_{k l}(x)=0$ for $k \neq l$. Note that $\left\{\Delta_{k l}(x)\right.$ : $\left.k, l \in \mathbb{S}, x \in \mathbb{R}^{d}\right\}$ are disjoint intervals and that the length of the interval $\Delta_{k l}(x)$ is equal to $q_{k l}(x)$. Define a function $h: \mathbb{R}^{d} \times \mathbb{S} \times \mathbb{R}_{+} \rightarrow \mathbb{R}$ by

$$
h(x, k, r)=\sum_{l \in \mathbb{S} \backslash\{k\}} 1_{\Delta_{k l}(x)}(r) .
$$

In other words, we set

$$
h(x, k, r)= \begin{cases}l-k & \text { if } r \in \Delta_{k l}(x) \\ 0 & \text { otherwise }\end{cases}
$$


for each $x \in \mathbb{R}^{d}$ and $k \in \mathbb{S}$. As a result, the process $\Lambda$ can be described as a solution to the following stochastic differential equation

$$
\Lambda(t)=\Lambda(0)+\int_{0}^{t} \int_{\mathbb{R}_{+}} h\left(X\left(s^{-}\right), \Lambda\left(s^{-}\right), r\right) N_{1}(d s, d r),
$$

where $N_{1}$ is a Poisson random measure on $[0, \infty) \times[0, \infty)$ with characteristic measure $\mathfrak{m}(d z)$, the Lebesgue measure.

We make the following standing assumption throughout the paper:

Assumption 1.1. For any $(x, k) \in \mathbb{R}^{d} \times \mathbb{S}$, the system of stochastic differential equations (1.1) and (1.4) has a non-explosive weak solution $\left(X^{(x, k)}, \Lambda^{(x, k)}\right)$ with initial condition $(x, k)$ and the solution is unique in the sense of probability law.

Consequently we can consider the semigroup

$$
P_{t} f(x, k):=\mathbb{E}_{x, k}[f(X(t), \Lambda(t))]=\mathbb{E}\left[f\left(X^{(x, k)}(t), \Lambda^{(x, k)}(t)\right)\right], \quad f \in \mathfrak{B}_{b}\left(\mathbb{R}^{d} \times \mathbb{S}\right) .
$$

The main focus of this paper is to investigate the continuity properties of the semigroup $P_{t}$. We say that the semigroup $P_{t}$ or the process $(X, \Lambda)$ is Feller continuous if $P_{t} f \in C_{b}\left(\mathbb{R}^{d} \times \mathbb{S}\right)$ for all $t \geq 0$ and $\lim _{t \downarrow 0} P_{t} f(x, k)=f(x, k)$ for all $f \in C_{b}\left(\mathbb{R}^{d} \times \mathbb{S}\right)$ and $(x, k) \in \mathbb{R}^{d} \times \mathbb{S}$. Furthermore, we say that the semigroup $P_{t}$ or the process $(X, \Lambda)$ is strong Feller continuous if $P_{t} f \in C_{b}\left(\mathbb{R}^{d} \times \mathbb{S}\right)$ for every $f \in \mathfrak{B}_{b}\left(\mathbb{R}^{d} \times \mathbb{S}\right)$ and $t>0$.

Denote the transition probability of the process $(X, \Lambda)$ by

$$
P(t,(x, k), B \times\{l\}):=P_{t} 1_{B \times\{l\}}(x, k)=\mathbb{P}\{(X(t), \Lambda(t)) \in B \times\{l\} \mid(X(0), \Lambda(0))=(x, k)\},
$$

for $B \in \mathfrak{B}\left(\mathbb{R}^{d}\right)$ and $l \in \mathbb{S}$. The semigroup $P_{t}$ of $(1.5)$ is said to be irreducible if for any $t>0$ and $(x, k) \in \mathbb{R}^{d} \times \mathbb{S}$, we have

$$
P(t,(x, k), B \times\{l\})>0
$$

for all $l \in \mathbb{S}$ and all nonempty open set $B \in \mathfrak{B}\left(\mathbb{R}^{d}\right)$.

For convenience, we state the infinitesimal generator of the regime-switching jump diffusion $(X, \Lambda)$ as follows:

$$
\mathscr{A} f(x, k):=\mathcal{L}_{k} f(x, k)+Q(x) f(x, k),
$$

for $f(\cdot, k) \in C_{c}^{2}\left(\mathbb{R}^{d}\right)$, where

$$
\begin{aligned}
\mathcal{L}_{k} f(x, k):= & \frac{1}{2} \operatorname{tr}\left(a(x, k) D^{2} f(x, k)\right)+\langle b(x, k), D f(x, k)\rangle \\
& +\int_{U}(f(x+c(x, k, u), k)-f(x, k)-\langle D f(x, k), c(x, k, u)\rangle) \nu(d u),
\end{aligned}
$$

and

$$
Q(x) f(x, k):=\sum_{l \in \mathbb{S}} q_{k l}(x)[f(x, l)-f(x, k)]=\int_{[0, \infty)}[f(x, k+h(x, k, z))-f(x, k)] \mathfrak{m}(d z) .
$$

In (1.7) and throughout the paper, $D f(x, k)$ and $D^{2} f(x, k)$ denote respectively the gradient and Hessian matrix of the function $f$ with respect to the $x$ variable, and $\langle\cdot, \cdot\rangle$ denotes the inner product. The HilbertSchmidt norm of a vector or a matrix $a$ is denoted by $|a|:=\sqrt{\operatorname{tr}\left(a a^{T}\right)}$, in which $a^{T}$ is the transpose of $a$. 


\subsection{Assumptions and Statements of Results}

We collect the assumptions and the main results in this subsection.

\subsubsection{Feller Property}

Assumption 1.2. (i) If $d=1$, then there exist a positive number $\delta_{0}$ and a nondecreasing and concave function $\rho:[0, \infty) \rightarrow[0, \infty)$ satisfying

$$
\int_{0^{+}} \frac{d r}{\rho(r)}=\infty
$$

such that for all $k \in \mathbb{S}, R>0$ and $x, z \in \mathbb{R}$ with $|x| \vee|z| \leq R$ and $|x-z| \leq \delta_{0}$,

$$
\begin{aligned}
& \operatorname{sgn}(x-z)(b(x, k)-b(z, k)) \leq \kappa_{R} \rho(|x-z|), \\
& |\sigma(x, k)-\sigma(z, k)|^{2}+\int_{U}|c(x, k, u)-c(z, k, u)|^{2} \nu(d u) \leq \kappa_{R}|x-z|,
\end{aligned}
$$

where $\kappa_{R}$ is a positive constant and $\operatorname{sgn}(a)=1_{\{a>0\}}-1_{\{a \leq 0\}}$. In addition, for each $k \in \mathbb{S}$, either

$$
\text { the function } x \mapsto x+c(x, k, u) \text { is nondecreasing for all } u \in U
$$

or there exists some $\beta>0$ such that

$$
|x-z+\theta(c(x, k, u)-c(z, k, u))| \geq \beta|x-z|, \forall(x, z, u, \theta) \in \mathbb{R} \times \mathbb{R} \times U \times[0,1] .
$$

(ii) If $d \geq 2$, then there exist a positive number $\delta_{0}$ and a nondecreasing and concave function $\rho:[0, \infty) \rightarrow[0, \infty)$ satisfying

$$
0<\rho(r) \leq(1+r)^{2} \rho(r /(1+r)) \text { for } r>0 \text { and } \int_{0^{+}} \frac{d r}{\rho(r)}=\infty
$$

so that for all $k \in \mathbb{S}, R>0$ and $x, z \in \mathbb{R}^{d}$ with $|x| \vee|z| \leq R$ and $|x-z| \leq \delta_{0}$,

$$
\begin{aligned}
2\langle x-z, b(x, k)-b(z, k)\rangle & +|\sigma(x, k)-\sigma(z, k)|^{2} \\
& +\int_{U}|c(x, k, u)-c(z, k, u)|^{2} \nu(d u) \leq \kappa_{R} \rho\left(|x-z|^{2}\right),
\end{aligned}
$$

where $\kappa_{R}$ is a positive constant.

Assumption 1.3. For each $k \in \mathbb{S}$, there exists a concave function $\gamma_{k}: \mathbb{R}_{+} \mapsto \mathbb{R}_{+}$with $\gamma(0)=0$ such that for all $x, y \in \mathbb{R}^{d}$ with $|x| \vee|y| \leq R$, we have

$$
\sum_{l \in \mathbb{S} \backslash\{k\}}\left|q_{k l}(x)-q_{k l}(y)\right| \leq \kappa_{R} \gamma_{k}(|x-y|) .
$$

for some positive constant $\kappa_{R}$ (which, without loss of generality, can be assumed to be the same positive constant as in that (1.10) and (1.11)).

Theorem 1.4. Under Assumptions 1.2 and 1.3, the process $(X, \Lambda)$ has the Feller property. 
Remark 1.5. We note that Assumption 1.2 is comparable to the corresponding assumption in $\mathrm{Xi}$, Yin \& Zhu (2019), except that the non-local term in (1.11) and (1.15) only requires the regularity of $\int_{U}|c(x, k, u)-c(z, k, u)|^{2} \nu(d u)$. In $\mathrm{Xi}$, Yin \& Zhu (2019), the corresponding term is $\int_{U}\left[|c(x, k, u)-c(z, k, u)|^{2} \wedge|x-z| \cdot|c(x, k, u)-c(z, k, u)|\right] \nu(d u)$.

Assumption 1.3 is weaker than that in Xi, Yin \& Zhu (2019). Indeed, the paper assumes that $Q(x)=\left(q_{k l}(x)\right)$ satisfies

$$
\sum_{l \in \mathbb{S} \backslash\{k\}}\left|q_{k l}(x)-q_{k l}(y)\right| \leq \kappa_{R} \rho\left(\frac{|x-y|}{1+|x-y|}\right), \text { for each } k \in \mathbb{S},
$$

for all $x, y \in \mathbb{R}^{d}$ with $|x| \vee|y| \leq R$, in which $\kappa_{R}>0$ and $\rho$ is an increasing and concave function satisfying (1.14). In contrast, the function $\gamma_{k}$ in Assumption 1.3 may depend on $k$, and is only required to be concave with $\gamma_{k}(0)=0$. In particular, the non-integrability condition $\int_{0^{+}} \frac{d r}{\rho(r)}=\infty$ is dropped. This relaxation is significant and renders that the analyses in Xi, Yin \& Zhu (2019) are not applicable.

\subsubsection{Strong Feller Property}

Assumption 1.6. For every $k \in \mathbb{S}$ the following assertions hold:

(i) For every $R>0$ there exits a constant $\lambda_{R}>0$ such that

$$
\langle\xi, a(x, k) \xi\rangle \geq \lambda_{R}|\xi|^{2}, \quad \xi \in \mathbb{R}^{d}
$$

for all $x \in \mathbb{R}^{d}$ with $|x| \leq R$, where $a(x, k):=\sigma(x, k) \sigma(x, k)^{T}$.

(ii) There exist a positive constant $\delta_{0}$ and a nonnegative function $g \in C(0, \infty)$ satisfying

$$
\int_{0}^{1} g(r) d r<\infty
$$

such that for each $R>0$, there exists a constant $\kappa_{R}>0$ so that either (a) or (b) below holds:

(a) If $d=1$, then

$$
2\langle x-z, b(x, k)-b(z, k)\rangle+\int_{U}|c(x, k, u)-c(z, k, u)|^{2} \nu(d u) \leq 2 \kappa_{R}|x-z| g(|x-z|),
$$

for all $x, z \in \mathbb{R}$ with $|x| \vee|z| \leq R$ and $|x-z| \leq \delta_{0}$.

(b) If $d \geq 2$, then

$$
\begin{aligned}
\mid \sigma_{\lambda_{R}}(x, k) & -\left.\sigma_{\lambda_{R}}(z, k)\right|^{2}+2\langle x-z, b(x, k)-b(z, k)\rangle \\
& +\int_{U}|c(x, k, u)-c(z, k, u)|^{2} \nu(d u) \leq 2 \kappa_{R}|x-z| g(|x-z|),
\end{aligned}
$$

for all $x, z \in \mathbb{R}^{d}$ with $|x| \vee|z| \leq R$ and $|x-z| \leq \delta_{0}$, where $\sigma_{\lambda_{R}}$ is the unique symmetric nonnegative definite matrix-valued function such that $\sigma_{\lambda_{R}}^{2}(x, k)=a(x, k)-\lambda_{R} I$.

Theorem 1.7. Suppose that Assumptions 1.3 and 1.6 hold. Then the process $(X, \Lambda)$ has strong Feller property. 
Remark 1.8. We remark that Assumption 1.6 improves significantly over those in the literature such as Shao (2015b), Xi \& Zhu (2017), which require Lipschitz condition for the coefficients of the associated stochastic differential equations. By contrast, (1.19) and (1.20) place very mild conditions on the coefficients. It allows us to treat, for example, the case of Hölder continuous coefficients by taking $g(r)=r^{-p}$ for $0 \leq p<1$; see Example 5.1. For the case when $d=1$, only the drift and the jump coefficients are required to satisfy the regularity conditions.

\subsubsection{Irreducibility}

Assumption 1.9. For each $k \in \mathbb{S}$ and $x \in \mathbb{R}^{d}$, the SDE

$$
X^{(k)}(t)=x+\int_{0}^{t} b\left(X^{(k)}(s), k\right) d s+\int_{0}^{t} \sigma\left(X^{(k)}(s), k\right) d W(s)+\int_{0}^{t} \int_{U} c\left(X^{(k)}(s-), k, u\right) \tilde{N}(d s, d u)
$$

has a non-explosive weak solution $X^{(k)}$ with initial condition $x$ and the solution is unique in the sense of probability law.

Assumption 1.10. For any $x \in \mathbb{R}^{d}$ and $k \in \mathbb{S}$, we have

$$
2\langle x, b(x, k)\rangle \leq \kappa\left(|x|^{2}+1\right), \quad|\sigma(x, k)|^{2}+\int_{U}|c(x, k, u)|^{2} \nu(d u) \leq \kappa\left(|x|^{2}+1\right),
$$

and

$$
\langle\xi, a(x, k) \xi\rangle \geq \lambda|\xi|^{2}, \quad \xi \in \mathbb{R}^{d}
$$

where $\lambda$ and $\kappa$ are positive constants.

Assumption 1.11. (i) There exists a positive constant $\kappa_{0}$ such that

$$
0 \leq q_{k l}(x) \leq \kappa_{0} l 3^{-l}
$$

for all $x \in \mathbb{R}^{d}$ and $k \neq l \in \mathbb{S}$.

(ii) For any $k, l \in \mathbb{S}$, there exist $k_{0}, k_{1}, \ldots, k_{n} \in \mathbb{S}$ with $k_{i} \neq k_{i+1}, k_{0}=k$, and $k_{n}=l$ such that the set $\left\{x \in \mathbb{R}^{d}: q_{k_{i} k_{i+1}}(x)>0\right\}$ has positive Lebesgue measure for all $i=0,1, \ldots, n-1$.

Theorem 1.12. Suppose that Assumptions 1.6 (ii), 1.9, 1.10, and 1.11 hold. Then the semigroup $P_{t}$ of (1.5) is irreducible.

Remark 1.13. As we mentioned in Remark 1.8, Assumption 1.6 (ii) places very mild regularity condition for the coefficients of the stochastic differential equations given by (1.1) and (1.4); see Remark 4.4 for further elaborations. Assumption 1.9 requires each subsystem of (1.1) to be wellposed in the weak sense. Assumption 1.6 (i) is strengthened to uniform ellipticity in (1.23), which is a common assumption in the literature for deriving irreducibility of (jump) diffusions, see, for example, Peszat \& Zabczyk (1995), Qiao (2014) and others. In addition, since we are dealing with a two-component process $(X, \Lambda)$, one can expect that the $q$-matrix $Q(x)$ must satisfy some sort of irreducibility condition so that $(X, \Lambda)$ is irreducible; Assumption 1.11 (ii) is therefore in force. Finally, the linear growth condition (1.22) as well as (1.24) are imposed to facilitate our technical analyses. 


\section{Feller Property}

This section is devoted to establishing the Feller property for regime-switching jump diffusion stated in Theorem 1.4. We will use the coupling method to prove Theorem 1.4. To this end, let us first construct a basic coupling operator $\mathscr{A}$ for $\mathscr{A}$. For $f(x, i, z, j) \in C_{c}^{2}\left(\mathbb{R}^{d} \times \mathbb{S} \times \mathbb{R}^{d} \times \mathbb{S}\right)$, we define

$$
\widetilde{\mathscr{A}} f(x, i, z, j):=\left[\widetilde{\Omega}_{\mathrm{d}}+\widetilde{\Omega}_{\mathrm{j}}+\widetilde{\Omega}_{\mathrm{s}}\right] f(x, i, z, j),
$$

where $\widetilde{\Omega}_{\mathrm{d}}, \widetilde{\Omega}_{\mathrm{j}}$, and $\widetilde{\Omega}_{\mathrm{S}}$ are defined as follows. For $x, z \in \mathbb{R}^{d}$ and $i, j \in \mathbb{S}$, we set $a(x, i)=\sigma(x, i) \sigma(x, i)^{\prime}$ and

$$
a(x, i, z, j)=\left(\begin{array}{cc}
a(x, i) & \sigma(x, i) \sigma(z, j)^{\prime} \\
\sigma(z, j) \sigma(x, i)^{\prime} & a(z, j)
\end{array}\right), \quad b(x, i, z, j)=\left(\begin{array}{l}
b(x, i) \\
b(z, j)
\end{array}\right) .
$$

Then we define

$$
\begin{aligned}
\widetilde{\Omega}_{\mathrm{d}} f(x, i, z, j):= & \frac{1}{2} \operatorname{tr}\left(a(x, i, z, j) D^{2} f(x, i, z, j)\right)+\langle b(x, i, z, j), D f(x, i, z, j)\rangle, \\
\widetilde{\Omega}_{\mathrm{j}} f(x, i, z, j):= & \int_{U}[f(x+c(x, i, u), i, z+c(z, j, u), j)-f(x, i, z, j) \\
& \left.-\left\langle D_{x} f(x, i, z, j), c(x, i, u)\right\rangle-\left\langle D_{z} f(x, i, z, j), c(z, j, u)\right\rangle\right] \nu(d u),
\end{aligned}
$$

where $D f(x, i, z, j)=\left(D_{x} f(x, i, z, j), D_{z} f(x, i, z, j)\right)^{\prime}$ is the gradient and $D^{2} f(x, i, z, j)$ is the Hessian matrix of $f$ with respect to the variables $x$ and $z$, and

$$
\begin{aligned}
\widetilde{\Omega}_{\mathrm{S}} f(x, i, z, j):= & \sum_{l \in \mathbb{S}}\left[q_{i l}(x)-q_{j l}(z)\right]^{+}(f(x, l, z, j)-f(x, i, z, j)) \\
& +\sum_{l \in \mathbb{S}}\left[q_{j l}(z)-q_{i l}(x)\right]^{+}(f(x, i, z, l)-f(x, i, z, j)) \\
& +\sum_{l \in \mathbb{S}}\left[q_{i l}(x) \wedge q_{j l}(z)\right](f(x, l, z, l)-f(x, i, z, j)) .
\end{aligned}
$$

For any function $f: \mathbb{R}^{d} \times \mathbb{R}^{d} \mapsto \mathbb{R}$, let $\tilde{f}: \mathbb{R}^{d} \times \mathbb{S} \times \mathbb{R}^{d} \times \mathbb{S} \mapsto \mathbb{R}$ be defined by $\tilde{f}(x, i, z, j):=f(x, z)$. Now we denote for each $k \in \mathbb{S}$

$$
\widetilde{\mathcal{L}}_{k} f(x, z)=\left(\widetilde{\Omega}_{\mathrm{d}}^{(k)}+\widetilde{\Omega}_{\mathrm{j}}^{(k)}\right) f(x, z):=\left(\widetilde{\Omega}_{\mathrm{d}}+\widetilde{\Omega}_{\mathrm{j}}\right) \widetilde{f}(x, k, z, k), \quad \forall f \in C_{c}^{2}\left(\mathbb{R}^{d} \times \mathbb{R}^{d}\right) .
$$

We introduce the following notations. Let $(X(\cdot), \Lambda(\cdot), \tilde{X}(\cdot), \tilde{\Lambda}(\cdot))$ denote the coupling process corresponding to the operator $\widetilde{\mathscr{A}}$ with initial condition $(x, k, z, k)$, in which $\delta_{0}>|x-z|>0$, and $\delta_{0}$ is the positive constant in Assumption 1.2. For any $R>0$, let

$$
\tau_{R}:=\inf \{t \geq 0:|\tilde{X}(t)| \vee|X(t)| \vee|\tilde{\Lambda}(t)| \vee|\Lambda(t)|>R\}
$$

In view of Assumption 1.1, $\lim _{R \rightarrow \infty} \tau_{R}=\infty$ a.s. Also denote $\Delta_{t}=\tilde{X}(t)-X(t)$ and

$$
S_{\delta_{0}}:=\inf \left\{t \geq 0:\left|\Delta_{t}\right|>\delta_{0}\right\}=\inf \left\{t \geq 0:|\tilde{X}(t)-X(t)|>\delta_{0}\right\} .
$$

Note that $\Lambda(0)=\tilde{\Lambda}(0)=k$. We denote by

$$
\zeta:=\inf \{t \geq 0: \Lambda(t) \neq \tilde{\Lambda}(t)\}
$$

the first time when the switching components $\Lambda$ and $\tilde{\Lambda}$ differ.

We need the following lemma whose proof is arranged in Appendix A: 
Lemma 2.1. Under Assumption 1.2, the following assertion holds:

$$
\lim _{|\tilde{x}-x| \rightarrow 0} \mathbb{E}\left[\left|\Delta_{t \wedge \tau_{R} \wedge S_{\delta_{0}} \wedge \zeta}\right|\right]=0, \quad \forall t \geq 0 .
$$

Proof of Theorem 1.4. We need to show that for each $(x, k) \in \mathbb{R}^{d} \times \mathbb{S}$ and each $f \in C_{b}\left(\mathbb{R}^{d} \times \mathbb{S}\right)$, the limit $\left(P_{t} f\right)(\tilde{x}, \tilde{k}) \rightarrow\left(P_{t} f\right)(x, k)$ as $(\tilde{x}, \tilde{k}) \rightarrow(x, k)$ holds for all $t \geq 0$. Since $\mathbb{S}=\{1,2, \ldots\}$ has a discrete topology, it is enough to consider only $(\tilde{x}, k) \rightarrow(x, k)$. First, observe that

$$
\begin{aligned}
\left|\left(P_{t} f\right)(\tilde{x}, \tilde{k})-\left(P_{t} f\right)(x, k)\right|= & |\mathbb{E}[f(\tilde{X}(t), \tilde{\Lambda}(t))]-\mathbb{E}[f(X(t), \Lambda(t))]| \\
\leq & |\mathbb{E}[f(\tilde{X}(t), \tilde{\Lambda}(t))]-\mathbb{E}[f(\tilde{X}(t), \Lambda(t))]| \\
& +|\mathbb{E}[f(\tilde{X}(t), \Lambda(t))]-\mathbb{E}[f(X(t), \Lambda(t))]| \\
= & \left|\mathbb{E}\left[(f(\tilde{X}(t), \tilde{\Lambda}(t))-f(\tilde{X}(t), \Lambda(t))) 1_{\{\zeta \leq t\}}\right]\right| \\
& +\left|\mathbb{E}\left[(f(\tilde{X}(t), \tilde{\Lambda}(t))-f(\tilde{X}(t), \Lambda(t))) 1_{\{\zeta>t\}}\right]\right| \\
& +|\mathbb{E}[f(\tilde{X}(t), \Lambda(t))]-\mathbb{E}[f(X(t), \Lambda(t))]| \\
\leq & 2|| f \|_{\infty} \mathbb{P}\{\zeta \leq t\}+|\mathbb{E}[f(\tilde{X}(t), \Lambda(t))]-\mathbb{E}[f(X(t), \Lambda(t))]| .
\end{aligned}
$$

We will show that both terms on the right-hand side of (2.9) converge to 0 as $\tilde{x} \rightarrow x$.

Consider the function $\Xi(x, k, z, l):=1_{\{k \neq l\}}$. It follows directly from the definition that

$$
\widetilde{\mathscr{A}} \Xi(x, k, z, l)=\widetilde{\Omega}_{\mathrm{s}} \Xi(x, k, z, l) \leq 0 \text {, if } k \neq l .
$$

When $k=l$, we have from (1.16) that

$$
\begin{aligned}
\widetilde{\mathscr{A} \Xi}(x, k, z, l) & =\widetilde{\Omega}_{\mathrm{s}} \Xi(x, k, z, k) \\
& =\sum_{i \in \mathbb{S}}\left[q_{k i}(x)-q_{k i}(z)\right]^{+}\left(1_{\{i \neq k\}}-1_{\{k \neq k\}}\right)+\sum_{i \in \mathbb{S}}\left[q_{k i}(z)-q_{k i}(x)\right]^{+}\left(1_{\{i \neq k\}}-1_{\{k \neq k\}}\right) \\
& \leq \sum_{i \in \mathbb{S}, i \neq k}\left|q_{k i}(x)-q_{k i}(z)\right| \leq \kappa_{R} \gamma_{k}(|x-y|) .
\end{aligned}
$$

Hence

$$
\widetilde{\mathscr{A}} \Xi(x, k, z, l) \leq \kappa_{R} \gamma_{k}(|x-y|)
$$

for all $k, l \in \mathbb{S}$ and $x, z \in \mathbb{R}^{d}$ with $|x| \vee|z| \leq R$.

Note that $\zeta \leq t \wedge \tau_{R} \wedge S_{\delta_{0}}$ if and only if $\tilde{\Lambda}\left(t \wedge \tau_{R} \wedge S_{\delta_{0}} \wedge \zeta\right) \neq \Lambda\left(t \wedge \tau_{R} \wedge S_{\delta_{0}} \wedge \zeta\right)$. Thus we can use (2.10) to compute

$$
\begin{aligned}
& \mathbb{P}\left\{\zeta \leq t \wedge \tau_{R} \wedge S_{\delta_{0}}\right\} \\
& =\mathbb{E}\left[\Xi\left(\tilde{X}\left(t \wedge \tau_{R} \wedge S_{\delta_{0}} \wedge \zeta\right), \tilde{\Lambda}\left(t \wedge \tau_{R} \wedge S_{\delta_{0}} \wedge \zeta\right), X\left(t \wedge \tau_{R} \wedge S_{\delta_{0}} \wedge \zeta\right), \Lambda\left(t \wedge \tau_{R} \wedge S_{\delta_{0}} \wedge \zeta\right)\right)\right] \\
& =\Xi(\tilde{x}, k, x, k)+\mathbb{E}\left[\int_{0}^{t \wedge \tau_{R} \wedge S_{\delta_{0}} \wedge \zeta} \widetilde{\mathscr{A}} \Xi(\tilde{X}(s), \tilde{\Lambda}(s), X(s), \Lambda(s)) d s\right] \\
& \leq \kappa_{R} \mathbb{E}\left[\int_{0}^{t \wedge \tau_{R} \wedge S_{\delta_{0}} \wedge \zeta} \gamma_{k}(|\tilde{X}(s)-X(s)|) d s\right] \\
& \leq \kappa_{R} \int_{0}^{t} \mathbb{E}\left[\gamma_{k}\left(\left|\tilde{X}\left(s \wedge \tau_{R} \wedge S_{\delta_{0}} \wedge \zeta\right)-X\left(s \wedge \tau_{R} \wedge S_{\delta_{0}} \wedge \zeta\right)\right|\right)\right] d s \\
& \leq \kappa_{R} \int_{0}^{t} \gamma_{k}\left(\mathbb { E } \left[\mid \Delta_{\left.\left.s \wedge \tau_{R} \wedge S_{\delta_{0}} \wedge \zeta \mid\right]\right) d s}\right.\right.
\end{aligned}
$$


where the last inequality follows from the concavity of $\gamma_{k}$. Since $\gamma_{k}(0)=0$, then $(2.8)$ and the bounded convergence theorem imply

$$
\lim _{|\widetilde{x}-x| \rightarrow 0} \mathbb{P}\left\{\zeta \leq t \wedge \tau_{R} \wedge S_{\delta_{0}}\right\}=0 .
$$

Note also that on the set $\left\{S_{\delta_{0}} \leq t \wedge \zeta \wedge \tau_{R}\right\}$ we have $\delta_{0} \leq\left|\Delta_{S_{\delta_{0}} \wedge t \wedge \zeta \wedge \tau_{R}}\right|$. This implies

$$
\delta_{0} \mathbb{P}\left\{S_{\delta_{0}} \leq t \wedge \zeta \wedge \tau_{R}\right\} \leq \mathbb{E}\left[\left|\Delta_{t \wedge S_{\delta_{0}} \wedge \zeta \wedge \tau_{R}}\right| 1_{\left\{S_{\delta_{0}} \leq t \wedge \zeta \wedge \tau_{R}\right\}}\right] \leq \mathbb{E}\left[\left|\Delta_{t \wedge S_{\delta_{0}} \wedge \zeta \wedge \tau_{R}}\right|\right] .
$$

Therefore, it follows from (2.8) that

$$
\lim _{|\widetilde{x}-x| \rightarrow 0} \mathbb{P}\left\{S_{\delta_{0}} \leq t \wedge \zeta \wedge \tau_{R}\right\}=0 .
$$

Fix an arbitrary positive number $\epsilon$. We have from (2.8) that

$$
\lim _{|\widetilde{x}-x| \rightarrow 0} \mathbb{P}\left\{\left|\Delta_{t \wedge S_{\delta_{0}} \wedge \tau_{R} \wedge \zeta}\right|>\epsilon\right\} \leq \lim _{|\widetilde{x}-x| \rightarrow 0} \frac{\mathbb{E}\left[\left|\Delta_{t \wedge S_{\delta_{0}} \wedge \tau_{R} \wedge \zeta}\right|\right]}{\epsilon}=0 .
$$

Since $\lim _{R \rightarrow \infty} \tau_{R}=\infty$ a.s., we can choose $R$ sufficiently large so that

$$
\mathbb{P}\left\{\tau_{R}<t\right\}<\epsilon .
$$

Then

$$
\begin{aligned}
\mathbb{P}\left\{\left|\Delta_{t}\right|>\varepsilon\right\}= & \mathbb{P}\left\{\left|\Delta_{t}\right|>\varepsilon, \tau_{R}<t\right\}+\mathbb{P}\left\{\left|\Delta_{t}\right|>\varepsilon, \tau_{R} \geq t, \zeta \leq t \wedge S_{\delta_{0}} \wedge \tau_{R}\right\} \\
& +\mathbb{P}\left\{\left|\Delta_{t}\right|>\varepsilon, \tau_{R} \geq t, \zeta>t \wedge S_{\delta_{0}} \wedge \tau_{R}, S_{\delta_{0}} \leq t \wedge \tau_{R} \wedge \zeta\right\} \\
& +\mathbb{P}\left\{\left|\Delta_{t}\right|>\varepsilon, \tau_{R} \geq t, \zeta>t \wedge S_{\delta_{0}} \wedge \tau_{R}, S_{\delta_{0}}>t \wedge \tau_{R} \wedge \zeta\right\} \\
\leq & \epsilon+\mathbb{P}\left\{\zeta \leq t \wedge S_{\delta_{0}} \wedge \tau_{R}\right\}+\mathbb{P}\left\{S_{\delta_{0}} \leq t \wedge \tau_{R} \wedge \zeta\right\}+\mathbb{P}\left\{\left|\Delta_{t}\right|>\varepsilon, t \leq S_{\delta_{0}} \wedge \tau_{R} \wedge \zeta\right\} \\
\leq & \epsilon+\mathbb{P}\left\{\zeta \leq t \wedge S_{\delta_{0}} \wedge \tau_{R}\right\}+\mathbb{P}\left\{S_{\delta_{0}} \leq t \wedge \tau_{R} \wedge \zeta\right\}+\mathbb{P}\left\{\left|\Delta_{t \wedge S_{\delta_{0}} \wedge \tau_{R} \wedge \zeta}\right|>\varepsilon\right\} .
\end{aligned}
$$

From (2.11)-(2.13) we have

$$
\lim _{|\widetilde{x}-x| \rightarrow 0} \mathbb{P}\left\{\left|\Delta_{t}\right|>\varepsilon\right\} \leq \epsilon .
$$

Since $\epsilon$ is arbitrary, we conclude that $\lim _{|\widetilde{x}-x| \rightarrow 0} \mathbb{P}\left\{\left|\Delta_{t}\right|>\varepsilon\right\}=0$. In other words, $\tilde{X}(t) \rightarrow X(t)$ in probability as $\tilde{x} \rightarrow x$. With the metric $d$ on $\mathbb{R}^{d} \times \mathbb{S}$ defined by $d((x, i),(y, j)):=|x-y|+1_{\{i \neq j\}}$, we see immediately that $(\tilde{X}(t), \Lambda(t)) \rightarrow(X(t), \Lambda(t))$ in probability as $\tilde{x} \rightarrow x$. Because the function $f$ is continuous, we also have $f(\tilde{X}(t), \Lambda(t)) \rightarrow f(X(t), \Lambda(t))$ in probability as $\tilde{x} \rightarrow x$. Then the bounded convergence theorem implies

$$
|\mathbb{E}[f(\tilde{X}(t), \Lambda(t))]-\mathbb{E}[f(X(t), \Lambda(t))]| \rightarrow 0 \text { as } \tilde{x} \rightarrow x .
$$

Next, we show that $\lim _{\tilde{x} \rightarrow x} \mathbb{P}\{\zeta \leq t\}=0$ holds. Thanks to (2.14) we can compute

$$
\begin{aligned}
\mathbb{P}\{\zeta \leq t\} & =\mathbb{P}\left\{\zeta \leq t, \tau_{R}<t\right\}+\mathbb{P}\left\{\zeta \leq t, \tau_{R} \geq t\right\} \\
& \leq \mathbb{P}\left\{\tau_{R}<t\right\}+\mathbb{P}\left\{\zeta \leq t, \tau_{R} \geq t, S_{\delta_{0}} \leq t \wedge \zeta\right\}+\mathbb{P}\left\{\zeta \leq t, \tau_{R} \geq t, S_{\delta_{0}}>t \wedge \zeta\right\} \\
& \leq \epsilon+\mathbb{P}\left\{S_{\delta_{0}} \leq t \wedge \zeta \wedge \tau_{R}\right\}+\mathbb{P}\left\{\zeta \leq t \wedge \tau_{R} \wedge S_{\delta_{0}}\right\} .
\end{aligned}
$$

It then follows from (2.11) and (2.12) that $\lim _{|\widetilde{x}-x| \rightarrow 0} \mathbb{P}\{\zeta \leq t\} \leq \epsilon$. Again since $\epsilon$ is arbitrary, we have

$$
\lim _{|\widetilde{x}-x| \rightarrow 0} \mathbb{P}\{\zeta \leq t\}=0
$$

Finally we plug (2.15) and (2.16) into (2.9) to complete the proof. 


\section{Strong Feller Property}

As in Section 2, we will use the coupling method to prove Theorem 1.7. To this end, we first define

$$
\widehat{a}(x, i, z, j):=\left(\begin{array}{cc}
a(x, i) & \hat{g}(x, i, z, j) \\
\hat{g}(x, i, z, j)^{T} & a(z, j)
\end{array}\right) \quad \text { and } \quad b(x, i, z, j):=\left(\begin{array}{c}
b(x, i) \\
b(z, j)
\end{array}\right)
$$

where

$$
\hat{g}(x, i, z, j):=\lambda_{R}\left(I-2 u(x, z) u(x, z)^{T}\right)+\sigma_{\lambda_{R}}(x, i) \sigma_{\lambda_{R}}(z, j)^{T},
$$

and $u(x, z):=\frac{x-z}{|x-z|}$. Then we define the coupling operator $\widehat{\mathscr{A}}$ for $\mathscr{A}$ of $(1.6)$ as follows:

$$
\widehat{\mathscr{A} f}(x, i, z, j):=\left[\widehat{\Omega}_{\mathrm{d}}+\widetilde{\Omega}_{\mathrm{j}}+\widetilde{\Omega}_{\mathrm{S}}\right] f(x, i, z, j), \quad f \in C_{c}^{2}\left(\mathbb{R}^{d} \times \mathbb{S} \times \mathbb{R}^{d} \times \mathbb{S}\right),
$$

where

$$
\widehat{\Omega}_{\mathrm{d}} f(x, i, z, j)=\frac{1}{2} \operatorname{tr}\left(\widehat{a}(x, i, z, j) D^{2} f(x, i, z, j)\right)+\langle b(x, i, z, j), D f(x, i, z, j)\rangle,
$$

and $\widetilde{\Omega}_{\mathrm{j}}$ and $\widetilde{\Omega}_{\mathrm{S}}$ are defined as in (2.3) and (2.4), respectively. In addition, as in Section 2, for each $k \in \mathbb{S}$ and any $F \in C_{c}^{2}\left(\mathbb{R}^{d} \times \mathbb{R}^{d}\right)$, we write $f(x, k, z, k):=F(x, z)$ and denote

$$
\widehat{\mathcal{L}}_{k} F(x, z)=\left[\widehat{\Omega}_{\mathrm{d}}^{(k)}+\widetilde{\Omega}_{\mathrm{j}}^{(k)}\right] f(x, k, z, k):=\widehat{\mathscr{A} f}(x, k, z, k) .
$$

Note that $\widehat{\mathcal{L}}_{k}$ is a coupling operator for $\mathcal{L}_{k}$ defined in (1.7).

Furthermore, to facilitate future presentations, we introduce the following notations. For any $x, z \in \mathbb{R}^{d}$ and $i, j \in \mathbb{S}$, we let

$$
\begin{aligned}
A(x, i, z, j) & :=a(x, i)+a(z, j)-2 \hat{g}(x, i, z, j), \\
\bar{A}(x, i, z, j) & :=\frac{1}{|x-z|^{2}}\langle x-z, A(x, i, z, j)(x-z)\rangle, \\
B(x, i, z, j) & :=\langle x-z, b(x, i)-b(z, j)\rangle .
\end{aligned}
$$

Lemma 3.1. For all $x, z \in \mathbb{R}^{d}$ and $i, j \in \mathbb{S}$, we have

(i) $\widehat{a}(x, i, z, j)$ is symmetric and uniformly positive definite,

(ii) $\operatorname{tr} A(x, i, z, j)=\left|\sigma_{\lambda_{R}}(x, i)-\sigma_{\lambda_{R}}(z, j)\right|^{2}+4 \lambda_{R}$, and

(iii) $\bar{A}(x, i, z, j) \geq 4 \lambda_{R}$.

Proof. The proof involves elementary and straightforward computations; similar computations can be found in Chen \& Li (1989) and Priola \& Wang (2006). We shall omit the details here.

Now, let $\phi \in C^{2}([0, \infty))$. As in Chen \& Li (1989), for each $k \in \mathbb{S}$ and all $x, z \in \mathbb{R}^{d}$ with $x \neq z$, we can verify that

$$
\begin{aligned}
\widehat{\Omega}_{\mathrm{d}}^{(k)} \phi(|x-z|)= & \frac{\phi^{\prime \prime}(|x-z|)}{2} \bar{A}(x, k, z, k) \\
& +\frac{\phi^{\prime}(|x-z|)}{2|x-z|}[\operatorname{tr} A(x, k, z, k)-\bar{A}(x, k, z, k)+2 B(x, k, z, k)] .
\end{aligned}
$$


Moreover, we have

$$
\begin{aligned}
\widetilde{\Omega}_{\mathrm{j}}^{(k)} \phi(|x-z|)= & \int_{U}(\phi(|x+c(x, k, u)-z-c(z, k, u)|)-\phi(|x-z|) \\
& \left.-\frac{\phi^{\prime}(|x-z|)}{|x-z|}\langle x-z, c(x, k, u)-c(z, k, u)\rangle\right) \nu(d u) .
\end{aligned}
$$

Motivated by Priola \& Wang (2006), we consider the function $G$ given by

$$
G(r):=\int_{0}^{r} \exp \left\{-\int_{0}^{s} \frac{\kappa_{R}}{2 \lambda_{R}} g(w) d w\right\} \int_{s}^{1} \exp \left\{\int_{0}^{v} \frac{\kappa_{R}}{2 \lambda_{R}} g(u) d u\right\} d v d s, \quad r \in[0,1],
$$

where $g$ is the function given in Assumption 1.6 (ii). Since $g \geq 0$, we see that

$$
G^{\prime}(r)=e^{-\int_{0}^{r} \frac{\kappa_{R}}{2 \lambda_{R}} g(w) d w} \int_{r}^{1} e^{\int_{0}^{v} \frac{\kappa_{R}}{2 \lambda_{R}} g(u) d u} d v \geq 0, \quad \text { and } \quad G^{\prime \prime}(r)=-1-\frac{\kappa_{R}}{2 \lambda_{R}} g(r) G^{\prime}(r) \leq 0 .
$$

Note also that $G$ is concave and $\lim _{r \rightarrow 0} G(r)=0$. Since $G^{\prime}(0) \geq 1$ and $G(0)=0$, there exists a constant $\alpha \in(0,1)$ so that

$$
r \leq G(r) \quad \text { for all } r \in[0, \alpha] .
$$

Lemma 3.2. Suppose that Assumptions 1.6 holds. Then for any $R>0$ and $k \in \mathbb{S}$ there exits a positive constant $\beta_{R}>0$ such that

$$
\widehat{\mathcal{L}}_{k} G(|x-z|) \leq-\beta_{R}
$$

for all $x, z \in \mathbb{R}^{d}$ with $|z| \vee|x| \leq R$ and $0<|x-z| \leq \alpha \wedge \delta_{0}$, where $\alpha>0$ is given in (3.7).

This lemma follows directly from straightforward but involved computations. To preserve the flow of reading, we arrange it to Appendix A.

Throughout the rest of the section, we use the following notations. For any $x, \tilde{x} \in \mathbb{R}^{d}$ and $k \in \mathbb{S}$, denote by $(X(\cdot), \Lambda(\cdot), \tilde{X}(\cdot), \tilde{\Lambda}(\cdot))$ the process corresponding to the coupling operator $\widehat{\mathscr{A}}$ with initial condition $(x, k, \tilde{x}, k)$. As in Section 2 , denote $\Delta_{t}:=\tilde{X}(t)-X(t)$ for $t \geq 0$. Let $\tau_{R}, S_{\delta_{0}}$, and $\zeta$ be defined as in (2.5), (2.6), and (2.7), respectively. In addition, for each $n \in \mathbb{N}$, we define

$$
T_{n}:=\inf \left\{t \geq 0:|X(t)-\tilde{X}(t)|<\frac{1}{n}\right\} .
$$

Then $\lim _{n \rightarrow \infty} T_{n}=T$, where

$$
T:=\inf \{t \geq 0: X(t)=\tilde{X}(t)\}
$$

Lemma 3.3. Suppose Assumption 1.6 holds. Then the following assertions hold for every $t \geq 0$ :

$$
\begin{aligned}
& \lim _{|\tilde{x}-x| \rightarrow 0} \mathbb{E}\left[G\left(\left|\Delta_{t \wedge \tau_{R} \wedge S_{\bar{\delta}} \wedge \zeta}\right|\right)\right]=0, \text { and } \\
& \lim _{|\tilde{x}-x| \rightarrow 0} \mathbb{E}\left[G\left(\left|\Delta_{t \wedge \tau_{R} \wedge S_{\bar{\delta}} \wedge \zeta^{-}}\right|\right)\right]=0 .
\end{aligned}
$$

In particular,

$$
\lim _{|\tilde{x}-x| \rightarrow 0} \mathbb{E}\left[\left|\Delta_{t \wedge \tau_{R} \wedge S_{\bar{\delta}} \wedge \zeta^{-}}\right|\right]=0,
$$

where $\bar{\delta}:=\delta_{0} \wedge \alpha, \delta_{0}$ is the constant given in Assumption 1.6 (ii), and $\alpha \in(0,1)$ is the constant given in (3.7). 
Proof. Assume without loss of generality that $\bar{\delta} \geq|x-\tilde{x}|>0$. We apply Itô's formula to the process $G(|\tilde{X}(\cdot)-X(\cdot)|)=G(|\Delta|$.$) :$

$$
\begin{aligned}
\mathbb{E}\left[G\left(\left|\Delta_{t \wedge \tau_{R} \wedge S_{\bar{\delta}} \wedge \zeta}\right|\right)\right] & =G\left(\left|\Delta_{0}\right|\right)+\mathbb{E}\left[\int_{0}^{t \wedge \tau_{R} \wedge S_{\bar{\delta}} \wedge \zeta} \widehat{\mathcal{L}} G\left(\left|\Delta_{s}\right|\right) d s\right] \\
& \leq G\left(\left|\Delta_{0}\right|\right)-\beta_{R} \mathbb{E}\left[t \wedge \tau_{R} \wedge S_{\bar{\delta}} \wedge \zeta\right]
\end{aligned}
$$

where the last inequality follows from (3.8). Hence

$$
\mathbb{E}\left[G\left(\left|\Delta_{t \wedge \tau_{R} \wedge S_{\bar{\delta}} \wedge \zeta}\right|\right)\right]+\beta_{R} \mathbb{E}\left[t \wedge \tau_{R} \wedge S_{\bar{\delta}} \wedge \zeta\right] \leq G\left(\left|\Delta_{0}\right|\right)=G(|x-\tilde{x}|) .
$$

Since $\lim _{r \rightarrow 0} G(r)=0$, (3.11) follows. The same argument implies (3.12). Since $\left|\Delta_{t \wedge \tau_{R} \wedge S_{\bar{\delta}} \wedge \zeta^{-}}\right| \leq$ $\bar{\delta} \leq \alpha$, it follows from (3.7) that

$$
\left|\Delta_{t \wedge \tau_{R} \wedge S_{\bar{\delta}} \wedge \zeta^{-}}\right| \leq G\left(\left|\Delta_{t \wedge \tau_{R} \wedge S_{\bar{\delta}} \wedge \zeta^{-}}\right|\right)
$$

and therefore (3.13) follows as well.

Lemma 3.4. Suppose that Assumptions 1.3 and 1.6 hold. Then

$$
\lim _{|\tilde{x}-x| \rightarrow 0} \mathbb{P}\{\zeta \leq t\}=0
$$

holds for every $t \geq 0$.

Proof. Given $\epsilon>0$. Choose $R$ sufficiently large so that $\mathbb{P}\left\{\tau_{R} \leq t\right\}<\epsilon$. Observe that

$$
\begin{aligned}
\mathbb{P}\{\zeta \leq t\} & =\mathbb{P}\left\{\zeta \leq t, \tau_{R}<t\right\}+\mathbb{P}\left\{\zeta \leq t, \tau_{R} \geq t\right\} \\
& \leq \mathbb{P}\left\{\tau_{R}<t\right\}+\mathbb{P}\left\{\zeta \leq t, \tau_{R} \geq t, S_{\bar{\delta}} \leq t \wedge \zeta\right\}+\mathbb{P}\left\{\zeta \leq t, \tau_{R} \geq t, S_{\bar{\delta}}>t \wedge \zeta\right\} \\
& \leq \epsilon+\mathbb{P}\left\{\zeta \leq t, \tau_{R} \geq t, S_{\bar{\delta}} \leq t \wedge \zeta\right\}+\mathbb{P}\left\{\zeta \leq t, \tau_{R} \geq t, S_{\bar{\delta}}>t \wedge \zeta\right\} \\
& \leq \epsilon+\mathbb{P}\left\{S_{\bar{\delta}} \leq t \wedge \zeta \wedge \tau_{R}\right\}+\mathbb{P}\left\{\zeta \leq t \wedge \tau_{R} \wedge S_{\bar{\delta}}\right\} .
\end{aligned}
$$

As in the proof of Theorem 1.4, condition (1.16) enables us to derive

$$
\mathbb{P}\left\{\zeta \leq t \wedge \tau_{R} \wedge S_{\bar{\delta}}\right\} \leq \kappa_{R} \int_{0}^{t} \gamma_{k}\left(\mathbb{E}\left[\left|\Delta_{s \wedge \tau_{R} \wedge S_{\bar{\delta}} \wedge \zeta^{-}}\right|\right]\right) d s .
$$

Furthermore, (3.13) implies that

$$
\lim _{|\tilde{x}-x| \rightarrow 0} \mathbb{P}\left\{\zeta \leq t \wedge \tau_{R} \wedge S_{\bar{\delta}}\right\}=0 .
$$

On the set $\left\{S_{\bar{\delta}} \leq t \wedge \zeta \wedge \tau_{R}\right\}$ we have $\bar{\delta} \leq\left|\Delta_{S_{\bar{\delta}} \wedge t \wedge \zeta \wedge \tau_{R}}\right|$. Since $G$ is increasing, we have

$$
0<G(\bar{\delta}) \leq G\left(\left|\Delta_{t \wedge S_{\bar{\delta}} \wedge \zeta \wedge \tau_{R}}\right|\right) .
$$

Thus

$$
G(\bar{\delta}) \mathbb{P}\left\{S_{\bar{\delta}} \leq t \wedge \zeta \wedge \tau_{R}\right\} \leq \mathbb{E}\left[G\left(\left|\Delta_{t \wedge S_{\bar{\delta}} \wedge \zeta \wedge \tau_{R}}\right|\right) 1_{\left\{S_{\bar{\delta}} \leq t \wedge \zeta \wedge \tau_{R}\right\}}\right] \leq \mathbb{E}\left[G\left(\left|\Delta_{t \wedge S_{\bar{\delta}} \wedge \zeta \wedge \tau_{R}}\right|\right)\right]
$$

This, together with (3.11), implies that

$$
\lim _{|\tilde{x}-x| \rightarrow 0} \mathbb{P}\left\{S_{\bar{\delta}} \leq t \wedge \zeta \wedge \tau_{R}\right\}=0 .
$$

In view of (3.15), it follows from (3.16) and (3.17) that $\lim _{|\tilde{x}-x| \rightarrow 0} \mathbb{P}\{\zeta \leq t\} \leq \epsilon$. Since $\epsilon$ is arbitrary, we obtain (3.14). 
Lemma 3.5. Suppose that Assumptions 1.3 and 1.6 hold. Then

$$
\lim _{|\tilde{x}-x| \rightarrow 0} \mathbb{P}\{t<T\}=0
$$

holds for every $t \geq 0$.

Proof. We may assume without loss of generality that $\bar{\delta} \geq|x-\tilde{x}|>\frac{1}{n_{0}}>0$ for some $n_{0} \in \mathbb{N}$. Let $\epsilon>0$ and choose a sufficiently large $R$ so that $\mathbb{P}\left\{\tau_{R} \leq t\right\}<\epsilon$. For each $n \geq n_{0}$, define $T_{n}$ and $T$ as in (3.9) and (3.10), respectively.

We first observe that

$$
\begin{aligned}
\mathbb{P}\{t<T\}= & \mathbb{P}\left\{t<T, \tau_{R}<t\right\}+\mathbb{P}\left\{t<T, \tau_{R} \geq t\right\} \\
\leq & \mathbb{P}\left\{\tau_{R}<t\right\}+\mathbb{P}\left\{t<T, \tau_{R} \geq t, S_{\bar{\delta}}<t\right\}+\mathbb{P}\left\{t<T, \tau_{R} \geq t, S_{\bar{\delta}} \geq t\right\} \\
\leq & \epsilon+\mathbb{P}\left\{S_{\bar{\delta}} \leq t \wedge T \wedge \tau_{R}\right\}+\mathbb{P}\left\{t \leq T \wedge \tau_{R} \wedge S_{\bar{\delta}}\right\} \\
= & \epsilon+\mathbb{P}\left\{S_{\bar{\delta}} \leq t \wedge T \wedge \tau_{R}, S_{\bar{\delta}} \leq \zeta\right\}+\mathbb{P}\left\{S_{\bar{\delta}} \leq t \wedge T \wedge \tau_{R}, S_{\bar{\delta}}>\zeta\right\} \\
& +\mathbb{P}\left\{t \leq T \wedge \tau_{R} \wedge S_{\bar{\delta}}, t<\zeta\right\}+\mathbb{P}\left\{t \leq T \wedge \tau_{R} \wedge S_{\bar{\delta}}, t \geq \zeta\right\} \\
\leq & \epsilon+\mathbb{P}\left\{S_{\bar{\delta}} \leq t \wedge T \wedge \tau_{R} \wedge \zeta\right\}+\mathbb{P}\left\{\zeta<S_{\bar{\delta}} \wedge t \wedge T \wedge \tau_{R}\right\} \\
& +\mathbb{P}\left\{t \leq T \wedge \tau_{R} \wedge S_{\bar{\delta}} \wedge \zeta\right\}+\mathbb{P}\{\zeta \leq t\} \\
\leq & \epsilon+\mathbb{P}\left\{S_{\bar{\delta}} \leq t \wedge T \wedge \tau_{R} \wedge \zeta\right\}+\mathbb{P}\{\zeta \leq t\}+\mathbb{P}\left\{t \leq T \wedge \tau_{R} \wedge S_{\bar{\delta}} \wedge \zeta\right\}+\mathbb{P}\{\zeta \leq t\} \\
= & \epsilon+\mathbb{P}\left\{S_{\bar{\delta}} \leq t \wedge T \wedge \tau_{R} \wedge \zeta\right\}+\mathbb{P}\left\{t \leq T \wedge \tau_{R} \wedge S_{\bar{\delta}} \wedge \zeta\right\}+2 \mathbb{P}\{\zeta \leq t\} \\
\leq & \epsilon+\mathbb{P}\left\{S_{\bar{\delta}} \leq T \wedge \tau_{R} \wedge \zeta\right\}+\frac{\mathbb{E}\left[T \wedge \tau_{R} \wedge S_{\bar{\delta}} \wedge \zeta\right]}{t}+2 \mathbb{P}\{\zeta \leq t\} .
\end{aligned}
$$

Note that on the set $\left\{S_{\bar{\delta}} \leq T_{n} \wedge \tau_{R} \wedge \zeta\right\}$ we have $\bar{\delta} \leq\left|\Delta_{S_{\bar{\delta}} \wedge T_{n} \wedge \tau_{R} \wedge \zeta}\right|$. Since $G$ is increasing, $0<G(\bar{\delta}) \leq G\left(\left|\Delta_{S_{\bar{\delta}} \wedge T_{n} \wedge \tau_{R} \wedge \zeta}\right|\right)$. Thus

$$
\begin{aligned}
G(\bar{\delta}) \mathbb{P}\left\{S_{\bar{\delta}} \leq T_{n} \wedge \tau_{R} \wedge \zeta\right\} & \leq \mathbb{E}\left[G\left(\left|\Delta_{S_{\bar{\delta}} \wedge T_{n} \wedge \tau_{R} \wedge \zeta}\right|\right) 1_{\left\{S_{\bar{\delta}} \leq T_{n} \wedge \tau_{R} \wedge \zeta\right\}}\right] \leq \mathbb{E}\left[G \left(\mid \Delta_{\left.\left.S_{\bar{\delta}} \wedge T_{n} \wedge \tau_{R} \wedge \zeta \mid\right)\right]}\right.\right. \\
& =G(|x-\tilde{x}|)+\mathbb{E}\left[\int_{0}^{S_{\bar{\delta}} \wedge T_{n} \wedge \tau_{R} \wedge \zeta} \widehat{\mathcal{L}}_{k} G\left(\left|\Delta_{s}\right|\right) d s\right] \\
& \leq G(|x-\tilde{x}|)-\beta_{R} \mathbb{E}\left[T_{n} \wedge \tau_{R} \wedge S_{\bar{\delta}} \wedge \zeta\right]
\end{aligned}
$$

where the last inequality follows from (3.8). So

$$
G(\bar{\delta}) \mathbb{P}\left\{S_{\bar{\delta}} \leq T_{n} \wedge \tau_{R} \wedge \zeta\right\}+\beta_{R} \mathbb{E}\left[T_{n} \wedge \tau_{R} \wedge S_{\bar{\delta}} \wedge \zeta\right] \leq G(|x-\tilde{x}|) .
$$

Passing to the limit as $n \rightarrow \infty$, we obtain

$$
G(\bar{\delta}) \mathbb{P}\left\{S_{\bar{\delta}} \leq T \wedge \tau_{R} \wedge \zeta\right\}+\beta_{R} \mathbb{E}\left[T \wedge \tau_{R} \wedge S_{\bar{\delta}} \wedge \zeta\right] \leq G(|x-\tilde{x}|) .
$$

Then, in view of (3.19), we have

$$
\begin{aligned}
\mathbb{P}\{t<T\} & \leq \epsilon+\mathbb{P}\left\{S_{\bar{\delta}} \leq T \wedge \tau_{R} \wedge \zeta\right\}+\frac{\mathbb{E}\left[T \wedge \tau_{R} \wedge S_{\bar{\delta}} \wedge \zeta\right]}{t}+2 \mathbb{P}\{\zeta \leq t\} \\
& \leq \epsilon+\frac{G(|x-\tilde{x}|)}{G(\bar{\delta})}+\frac{G(|x-\tilde{x}|)}{t \beta}+2 \mathbb{P}\{\zeta \leq t\}
\end{aligned}
$$

From (3.14) and the fact that $\lim _{|\tilde{x}-x| \rightarrow 0} G(|x-\tilde{x}|)=0$, we obtain $\lim _{|\tilde{x}-x| \rightarrow 0} \mathbb{P}\{t<T\} \leq \epsilon$. Since $\epsilon$ was arbitrary, we obtain (3.18). 
Now we are ready to present the proof of Theorem 1.7.

Proof of Theorem 1.7. Given $x \in \mathbb{R}^{d}$ and $k \in \mathbb{S}$. We want to show that for every $f \in \mathfrak{B}_{b}\left(\mathbb{R}^{d} \times \mathbb{S}\right)$, the limit $\left(P_{t} f\right)(\tilde{x}, \tilde{k}) \rightarrow\left(P_{t} f\right)(x, k)$ as $(\tilde{x}, \tilde{k}) \rightarrow(x, k)$ holds for all $t>0$. As in the proof of Theorem 1.4, we only need to consider the case when $\tilde{k}=k$.

For any given $\epsilon>0$ we can choose a sufficiently large $R$ so that $\mathbb{P}\left\{\tau_{R} \leq t\right\}<\epsilon$. Let $\tilde{x} \in \mathbb{R}^{d}$ be such that $\bar{\delta} \geq|x-\tilde{x}|>0$, where $\bar{\delta}:=\delta_{0} \wedge \alpha$. Denote the coupling process corresponding to the coupling operator $\widehat{\mathscr{A}}$ defined in (3.1) with initial condition $(x, k, \tilde{x}, k)$ by $(X(t), \Lambda(t), \tilde{X}(t), \tilde{\Lambda}(t))$. Denote by

$$
\tilde{T}:=\inf \{t \geq 0:(X(t), \Lambda(t))=(\tilde{X}(t), \tilde{\Lambda}(t))\}
$$

the coupling time of $(X(t), \Lambda(t))$ and $(\tilde{X}(t), \tilde{\Lambda}(t))$. Recall the stopping time $T$ defined in (3.10). We make the following observations: (i) $T \leq \tilde{T}$, and (ii) $T<\zeta$ implies $T=\tilde{T}$. We then have

$$
\begin{aligned}
1_{\{t<\tilde{T}\}} & =1_{\{t<T\}}+1_{\{T \leq t<\tilde{T}\}} \\
& =1_{\{t<T\}}+1_{\{T \leq t<\tilde{T}, \zeta \leq t\}}+1_{\{T \leq t<\tilde{T}, \zeta>t\}} \\
& \leq 1_{\{t<T\}}+1_{\{\zeta \leq t\}}+1_{\{T \leq t, t<\zeta, t<\tilde{T}\}} \\
& \leq 1_{\{t<T\}}+1_{\{\zeta \leq t\}}+1_{\{T<\zeta, t<\tilde{T}\}} \\
& \leq 1_{\{t<T\}}+1_{\{\zeta \leq t\}}+1_{\{T=\tilde{T}, t<\tilde{T}\}} \\
& \leq 1_{\{t<T\}}+1_{\{\zeta \leq t\}}+1_{\{t<T\}} \\
& =2 \cdot 1_{\{t<T\}}+1_{\{\zeta \leq t\}} .
\end{aligned}
$$

Then it follows that

$$
\begin{aligned}
\left|\left(P_{t} f\right)(\tilde{x}, \tilde{k})-\left(P_{t} f\right)(x, k)\right|= & |\mathbb{E}[f(\tilde{X}(t), \tilde{\Lambda}(t))]-\mathbb{E}[f(X(t), \Lambda(t))]| \\
\leq & \mathbb{E}\left[|f(\tilde{X}(t), \tilde{\Lambda}(t))-f(X(t), \Lambda(t))| 1_{\{t<\tilde{T}\}}\right] \\
& +\mathbb{E}\left[|f(\tilde{X}(t), \tilde{\Lambda}(t))-f(X(t), \Lambda(t))| 1_{\{t \geq \tilde{T}\}}\right] \\
= & \mathbb{E}\left[|f(\tilde{X}(t), \tilde{\Lambda}(t))-f(X(t), \Lambda(t))| 1_{\{t<\tilde{T}\}}\right] \\
\leq & 2\|f\|_{\infty} \mathbb{E}\left[1_{\{t<\tilde{T}\}}\right] \\
\leq & 2\|f\|_{\infty} \mathbb{E}\left[2 \cdot 1_{\{t<T\}}+1_{\{\zeta \leq t\}}\right] \\
= & 4\|f\|_{\infty} \mathbb{P}\{t<T\}+2\|f\|_{\infty} \mathbb{P}\{\zeta \leq t\} .
\end{aligned}
$$

A combination of (3.14) and (3.18) then gives

$$
\lim _{|\tilde{x}-x| \rightarrow 0}\left|\left(P_{t} f\right)(\tilde{x}, \tilde{k})-\left(P_{t} f\right)(x, k)\right|=0 .
$$

This establishes the strong Feller property and concludes the proof.

\section{Irreducibility}

This section aims to establish irreducibility for the process $(X, \Lambda)$. The general approach can be described as follows. We first show that for any $k \in \mathbb{S}$, the process $X^{(k)}$ of (1.21) is strong Feller and irreducible. Then we use a result in $\mathrm{Xi}$, Yin \& Zhu (2019) to write $P(t,(x, k), B \times\{l\})$ as a 
convergent series in terms of sub-transition probabilities of the killed processes $\tilde{X}^{(j)}, j \in \mathbb{S}$ and the transition rates $q_{j l}(x)$.

Denote the transition probability of the process $X^{(k)}$ by

$$
P^{(k)}(t, x, B):=\mathbb{P}\left\{X^{(k)}(t) \in B \mid X^{(k)}(0)=x\right\}, \quad B \in \mathfrak{B}\left(\mathbb{R}^{d}\right) .
$$

The corresponding semigroup $P_{t}^{(k)}$ is said to be irreducible if $P^{(k)}(t, x, B)>0$ for all nonempty open set $B \subset \mathbb{R}^{d}$. We next kill the process $X^{(k)}$ with killing rate $q_{k}(\cdot)$ and denote the killed process by $\tilde{X}^{(k)}$, that is, we define

$$
\tilde{X}^{(k)}(t)= \begin{cases}X^{(k)}(t) & \text { if } t<\tau, \\ \partial & \text { if } t \geq \tau,\end{cases}
$$

where $\tau:=\inf \{t \geq 0: \Lambda(t) \neq \Lambda(0)\}$ and $\partial$ is a cemetery point added to $\mathbb{R}^{d}$. Then the semigroup of the killed process $\tilde{X}^{(k)}$ is given by

$$
\tilde{P}_{t}^{(k)} f(x):=\mathbb{E}_{x}\left[f\left(\tilde{X}^{(k)}(t)\right)\right]=\mathbb{E}\left[f\left(X^{(k)}(t)\right) \exp \left\{\int_{0}^{t} q_{k k}\left(X^{(k)}(s)\right) d s\right\} \mid X^{(k)}(0)=x\right],
$$

where $f \in \mathfrak{B}_{b}\left(\mathbb{R}^{d}\right)$. We also denote its sub-transition probability by

$$
\tilde{P}^{(k)}(t, x, B):=\tilde{P}_{t}^{(k)} 1_{B}(x)=\mathbb{E}_{x}\left[1_{B}\left(\tilde{X}^{(k)}(t)\right)\right]=\mathbb{P}\left\{\tilde{X}^{(k)}(t) \in B \mid \tilde{X}^{(k)}(0)=x\right\}, \quad B \in \mathfrak{B}\left(\mathbb{R}^{d}\right) .
$$

Lemma 4.1. Under Assumptions 1.6 and 1.9, the semigroup $P_{t}^{(k)}$ is strong Feller.

Proof. Let $\left(\widetilde{X}^{(k)}, X^{(k)}\right)$ be the coupling process corresponding to $\widehat{\mathcal{L}}_{k}$ of $(3.3)$ with initial condition $(\tilde{x}, x)$. Suppose without loss of generality that $0<|\tilde{x}-x|<\delta_{0}$, where $\delta_{0}$ is the positive constant in Assumption 1.6. Define $T:=\inf \{t \geq 0: \widetilde{X}(t)=X(t)\}$. Using very similar calculations as those in the proof of Lemma 3.5, we can show that $\lim _{|\tilde{x}-x| \rightarrow 0} \mathbb{P}\{t<T\}=0$. Then, for any $f \in \mathfrak{B}_{b}\left(\mathbb{R}^{d}\right)$ and $t>0$, we have

$$
\left|\left(P_{t}^{(k)} f\right)(\tilde{x})-\left(P_{t}^{(k)} f\right)(x)\right|=\left|\mathbb{E}\left[f\left(\tilde{X}^{(k)}(t)\right)\right]-\mathbb{E}\left[f\left(X^{(k)}(t)\right)\right]\right| \leq 2\|f\|_{\infty} \mathbb{P}\{t<T\} \rightarrow 0,
$$

as $\tilde{x}-x \rightarrow 0$. This implies that $P_{t}^{(k)} f$ is a continuous function and hence completes the proof.

Lemma 4.2. Suppose that Assumption 1.10 holds. Then for every $T>0$ there exists a constant $K:=K(T, X(0))>0$ so that

$$
\mathbb{E}\left[|X(t)|^{2}\right] \leq K
$$

for all $t \in[0, T]$.

Proof. This lemma follows from (1.22) and standard arguments. For brevity, we omit the details here.

To derive irreducibility for the semigroup $P_{t}^{(k)}$, we consider the function $F$ given by

$$
F(r):=\int_{0}^{\frac{r}{1+r}} e^{-\int_{0}^{s} g(w) d w} d s, \quad r \in[0, \infty)
$$

where $g$ is the function given in Assumption 1.6(ii). Since $g \geq 0$, we see that

$$
0 \leq F(r) \leq \frac{r}{1+r} \leq 1
$$




$$
0 \leq F^{\prime}(r)=\frac{1}{(1+r)^{2}} e^{-\int_{0}^{\frac{r}{1+r}} g(w) d w} \leq \frac{1}{(1+r)^{2}} \leq 1
$$

and

$$
0 \geq F^{\prime \prime}(r)=-\left[\frac{2}{1+r}+\frac{g\left(\frac{r}{1+r}\right)}{(1+r)^{2}}\right] F^{\prime}(r)
$$

In addition, for any $x \in \mathbb{R}^{d}$, we have

$$
\nabla F\left(|x|^{2}\right)=2 F^{\prime}\left(|x|^{2}\right) x, \quad \nabla^{2} F\left(|x|^{2}\right)=4 F^{\prime \prime}\left(|x|^{2}\right) x x^{T}+2 F^{\prime}\left(|x|^{2}\right) I .
$$

Lemma 4.3. Under Assumptions 1.1, 1.6 (ii), and 1.10, the semigroup $P_{t}^{(k)}$ is irreducible.

Remark 4.4. While irreducibility for jump diffusions has been considered in the literature such as Qiao (2014), Xi \& Zhu (2019), it is worth pointing out that Assumption 1.6(ii) is much weaker than Assumptions $\left(\mathrm{H}_{1}^{\prime}\right)$ and $\left(\mathrm{H}_{f}^{\prime}\right)$ of Qiao (2014) and Assumption 2.5 of Xi \& Zhu (2019). In particular, as we mentioned in Remark 1.8, Assumption 1.6(ii) allows to treat SDEs with merely Hölder continuous coefficients. The relaxations make the analyses more involved and subtle than those in the literature. Nevertherless, to preserve the flow of reading, we defer the proof of Lemma 4.3 to Appendix A.

Proof of Theorem 1.12. Given $t>0$ and $(x, k) \in \mathbb{R}^{d} \times \mathbb{S}$. We want to show that $P(t,(x, k), B \times$ $\{l\})>0$ for all $l \in \mathbb{S}$ and all $B \in \mathfrak{B}\left(\mathbb{R}^{d}\right)$ with positive Lebesgue measure. Under Assumption 1.11 and from Lemma 4.1, as in the proof of Theorem 4.8 of Xi, Yin \& Zhu (2019), we can write

$$
\begin{aligned}
& P(t,(x, k), B \times\{l\}) \\
& =\delta_{k l} \tilde{P}^{(k)}(t, x, B)+\sum_{m=1}^{\infty} \int_{0<t_{1}<\cdots<t_{m}<t} \cdots \int_{\substack{l_{0}, l_{1}, l_{2}, \ldots, l_{m} \in \mathbb{S} \\
l_{i} \neq l_{i+1}, l_{0}=k, l_{m}=l^{\mathbb{R}^{d}}}} \cdots \int_{\mathbb{R}^{d}} \tilde{P}^{\left(l_{0}\right)}\left(t_{1}, x, d y_{1}\right) q_{l_{0} l_{1}}\left(y_{1}\right) \\
& \quad \times \tilde{P}^{\left(l_{1}\right)}\left(t_{2}-t_{1}, y_{1}, d y_{2}\right) \cdots q_{l_{m-1} l_{m}}\left(y_{m}\right) \tilde{P}^{\left(l_{m}\right)}\left(t-t_{m}, y_{m}, B\right) d t_{1} d t_{2} \cdots d t_{m},
\end{aligned}
$$

where $\delta_{k l}$ is the Kronecker symbol. From Assumption 1.11 (ii), we know that the set $\left\{y \in \mathbb{R}^{d}\right.$ : $\left.q_{l_{i} l_{i+1}}(y)>0\right\}$ has positive Lebesgue measure. Then it suffices to show that $\tilde{P}^{(k)}(s, y, B)>0$ for all $k \in \mathbb{S}, s>0$ and $B \in \mathfrak{B}\left(\mathbb{R}^{d}\right)$. We calculate

$$
\begin{aligned}
\tilde{P}^{(k)}(s, y, B) & =\mathbb{P}\left\{\tilde{X}_{y}^{(k)}(s) \in B\right\}=\mathbb{E}_{k}\left[1_{B}\left(X_{y}^{(k)}(s)\right) \exp \left(-\int_{0}^{s} q_{k}\left(X_{y}^{(k)}(r)\right) d r\right)\right] \\
& \geq \mathbb{E}_{k}\left[1_{B}\left(X_{y}^{(k)}(s)\right) e^{-M}\right] \geq e^{-M} \mathbb{P}\left\{X_{y}^{(k)}(s) \in B\right\}=e^{-M} P^{(k)}(s, y, B) .
\end{aligned}
$$

From Lemma 4.3, the semigroup associated with the process $X^{(k)}$ is irreducible and therefore $P^{(k)}(s, y, B)>0$. This completes the proof.

Proposition 4.5. Suppose that Assumptions 1.1, 1.2, and 1.3 hold. In addition, assume there exist constants $\alpha, \beta>0$, a compact subset $C \subset \mathbb{R}^{d}$, a compact subset $N \subset \mathbb{S}$, a measurable function $f: \mathbb{R}^{d} \times \mathbb{S} \rightarrow[1, \infty)$, and a twice continuously differentiable function $V: \mathbb{R}^{d} \times \mathbb{S} \rightarrow[0, \infty)$ such that

$$
\mathscr{A} V(x, k) \leq-\alpha f(x, k)+\beta 1_{C \times N}(x, k), \quad \forall(x, k) \in \mathbb{R}^{d} \times \mathbb{S} .
$$

Then the the semigroup $P_{t}$ of (1.5) has an invariant probability measure $\pi$. 
Proof. Since the proof is very similar to those in Xi (2004) or Xi \& Zhu (2019), we shall only give the sketch here. We first use (4.7) and Itô's formula to derive

$$
\alpha t \leq V(x, k)+\beta \int_{0}^{t} P(s,(x, k), C \times N) d s, \quad \forall t>0,
$$

which, in turn, implies that

$$
\liminf _{t \longrightarrow \infty} \frac{1}{t} \int_{0}^{t} P(s,(x, k), C \times N) d s \geq \frac{\alpha}{\beta}>0
$$

We have shown in Theorem 1.4 that the process $(X, \Lambda)$ is Feller under Assumptions 1.1-1.3. Then, in view of Foguel (1969) and Stettner (1986) (see also the proof of Theorem 4.5 of Meyn \& Tweedie (1993b)), (4.8) implies that an invariant measure $\pi$ exists.

Proposition 4.6. Suppose that Assumptions 1.1, 1.3, 1.6, 1.9, 1.10, and 1.11 hold. If there exists a twice continuously differentiable function $V: \mathbb{R}^{d} \times \mathbb{S} \rightarrow[0, \infty)$ such that (4.7) holds, then the the semigroup $P_{t}$ of (1.5) has a unique invariant measure.

Proof. The existence of an invariant measure follows directly from Proposition 4.5. For the uniqueness, we note that $P_{t}$ is strong Feller and irreducible by Theorems 1.7 and 1.12, respectively. Then by Cerrai (2001) and also Hairer (2016), $P_{t}$ can admit at most one invariant measure. This completes the proof.

\section{Examples}

Example 5.1. Consider the following SDE

$$
d X(t)=b(X(t), \Lambda(t)) d t+\sigma(X(t), \Lambda(t)) d W(t)+\int_{U} c\left(X\left(t^{-}\right), \Lambda\left(t^{-}\right), u\right) \tilde{N}(d t, d u)
$$

with initial condition $X(0)=x \in \mathbb{R}$, where $W$ is a standard 1-dimensional Brownian motion, $\tilde{N}$ is the associated compensated Poisson random measure on $[0, \infty) \times U$ with intensity $d t \nu(d u)$ in which $U=\{u \in \mathbb{R}: 0<|u|<1\}$ and $\nu(d u):=\frac{d u}{|u|^{2}}$. Note that $\nu$ is a $\sigma$-finite measure on $U$ with $\nu(U)=\infty$. The component $\Lambda$ is the continuous-time stochastic process taking values in $\mathbb{S}=\{1,2, \ldots\}$ generated by $Q(x)=\left(q_{k l}(x)\right)$ where

$$
q_{k l}(x)= \begin{cases}\frac{k}{3^{l+k}} \frac{1}{\left(1+l|x|^{2}\right)} & \text { if } k \neq l \\ -\sum_{l \neq k} q_{k l}(x) & \text { otherwise }\end{cases}
$$

Furthermore, suppose the coefficients of (5.1) are given by

$$
\sigma(x, k)=x^{\frac{2}{3}}+1, \quad b(x, k)=-\frac{x}{2 k^{2}}, \quad c(x, k, u)=\frac{u x}{\sqrt{2} k}, \quad \text { for }(x, k) \in \mathbb{R} \times \mathbb{S} \text { and } u \in U .
$$

We make the following observations.

(i) Assumption 1.1 is satisfied. Indeed, one can verify directly that the coefficients of (5.1) satisfy the linear growth condition and Assumption 2.2 of Xi, Yin \& Zhu (2019). By Theorem 2.5 of Xi, Yin \& Zhu (2019), (5.1) has a unique strong non-explosive solution. This, of course, 
implies Assumption 1.1. In addition, Assumption 1.10 holds. Indeed, for any $k \in \mathbb{S}$ and $x \in \mathbb{R}$, we have $2\langle x, b(x, k)\rangle=-\frac{x^{2}}{2 k^{2}}$ and

$$
\begin{aligned}
|\sigma(x, k)|^{2}+\int_{U}|c(x, k, u)|^{2} \nu(d u) & =\left(x^{\frac{2}{3}}+1\right)^{2}+\frac{|x|^{2}}{2 k^{2}} \int_{U}|u|^{2} \nu(d u) \\
& =\left(x^{\frac{2}{3}}+1\right)^{2}+\frac{|x|^{2}}{k^{2}} \\
& \leq 4\left[|x|^{2}+1\right] .
\end{aligned}
$$

Hence (1.22) holds with $\kappa=4$. Since $a(x, k)=\sigma^{2}(x, k)=\left(x^{\frac{2}{3}}+1\right)^{2} \geq 1$, (1.23) holds with $\lambda=1$.

(ii) It is clear that Assumption 1.2 (i) holds true. Next we verify Assumption 1.3. To this end, we compute

$$
\begin{aligned}
\sum_{l \in \mathbb{S} \backslash\{k\}}\left|q_{k l}(x)-q_{k l}(y)\right| & =\sum_{l \in \mathbb{S} \backslash\{k\}}\left|\frac{k}{3^{l+k}} \frac{1}{\left(1+l|x|^{2}\right)}-\frac{k}{3^{l+k}} \frac{1}{\left(1+l|y|^{2}\right)}\right| \\
& =\frac{k}{3^{k}} \sum_{l \in \mathbb{S} \backslash\{k\}} \frac{1}{3^{l}}\left|\frac{1}{1+l|x|^{2}}-\frac{1}{1+l|y|^{2}}\right| \\
& \leq \sum_{l \in \mathbb{S}} \frac{l}{3^{l}} \frac{\left.|| y\right|^{2}-|x|^{2} \mid}{\left(1+l|x|^{2}\right)\left(1+l|y|^{2}\right)} \\
& =\sum_{l \in \mathbb{S}} \frac{l}{3^{l}} \frac{(|y|+|x|)|| y|-| x||}{\left(1+l|x|^{2}\right)\left(1+l|y|^{2}\right)} \\
& \leq \sum_{l \in \mathbb{S}} \frac{l}{3^{l}}|y-x|=\frac{3}{4}|x-y|,
\end{aligned}
$$

where the last inequality follows from the triangle inequality ||$y|-| x|| \leq|x-y|$ and the observation that

$$
\frac{|y|+|x|}{\left(1+l|x|^{2}\right)\left(1+l|y|^{2}\right)} \leq \frac{|y|}{1+l|y|^{2}}+\frac{|x|}{1+l|x|^{2}} \leq \frac{|y|}{1+|y|^{2}}+\frac{|x|}{1+|x|^{2}} \leq \frac{1}{2}+\frac{1}{2}=1 .
$$

(iii) We can further verify that Assumption 1.6 holds. Indeed, since $a(x, k)=\sigma^{2}(x, k)=x^{\frac{4}{3}}+$ $2 x^{\frac{2}{3}}+1$, for each $R>0$, we can take $\lambda_{R}=1$ and $\sigma_{\lambda_{R}}(x, k)=\left(x^{\frac{4}{3}}+2 x^{\frac{2}{3}}\right)^{\frac{1}{2}}$ for all $(x, k) \in \mathbb{R} \times \mathbb{S}$. Then it is straightforward to verify that for all $x, z \in \mathbb{R}$ with $|x| \vee|z| \leq R$ and $k \in \mathbb{S}$

$$
\begin{aligned}
& \left|\sigma_{\lambda_{R}}(x, k)-\sigma_{\lambda_{R}}(z, k)\right|^{2}+2\langle x-z, b(x, k)-b(z, k)\rangle+\int_{U}|c(x, k, u)-c(z, k, u)|^{2} \nu(d u) \\
& \quad \leq 2\left(z^{\frac{2}{3}}+x^{\frac{2}{3}}+2\right)\left(z^{\frac{2}{3}}-x^{\frac{2}{3}}\right)-\frac{1}{k^{2}}|x-z|^{2}+\frac{1}{k^{2}}|x-z|^{2} \\
& \quad \leq 4\left(R^{\frac{2}{3}}+1\right)|x-z|^{\frac{2}{3}} \\
& \quad=4\left(R^{\frac{2}{3}}+1\right)|x-z| g(|x-z|),
\end{aligned}
$$

where $g(r)=r^{-\frac{1}{3}}$. Note that the function $g$ satisfies (1.18). As a result, (5.1) is strong Feller continuous by Theorem 1.7. 
(iv) Next we see immediately that Assumptions 1.11 holds and hence (5.1) is irreducible by virtue of Theorem 1.12.

Example 5.2. Consider the following SDE

$$
\begin{aligned}
d X(t) & =b(X(t), \Lambda(t)) d t+\sigma(X(t), \Lambda(t)) d W(t)+\int_{U} c\left(X\left(t^{-}\right), \Lambda\left(t^{-}\right), u\right) \tilde{N}(d t, d u), \\
X(0) & =x \in \mathbb{R}^{2}
\end{aligned}
$$

where $W$ is a standard 2-dimensional Brownian motion, $\tilde{N}$ is the associated compensated Poisson random measure on $[0, \infty) \times U$ with intensity $d t \nu(d u)$ in which $U=\left\{u \in \mathbb{R}^{2}: 0<|u|<1\right\}$ and $\nu(d u):=\frac{d u}{|u|^{2+\delta}}$ for some $\delta \in(0,2)$. The component $\Lambda$ is the continuous-time stochastic process taking values in $\mathbb{S}=\{1,2, \ldots\}$ generated by $Q(x)=\left(q_{k l}(x)\right)$ with $q_{k l}(x)=\frac{2+\cos (k|x|)}{3^{l}\left(2+\sin \left(|x|^{2}\right)\right)}$ for $x \in \mathbb{R}^{2}$ and $k \neq l \in \mathbb{S}$ and $q_{k}(x)=-q_{k k}(x)=\sum_{l \neq k} q_{k l}(x)$. The coefficients of (5.2) are given by

$$
\sigma(x, k)=\frac{|x|+1}{4} I, \quad b(x, k)=-\frac{k}{k+1} x, \quad c(x, k, u)=\frac{\sqrt{k}}{\sqrt{k+1}} \gamma|u| x
$$

where $I$ is the 2-dimensional identity matrix and $\gamma$ is a positive constant so that $\gamma^{2} \int_{U}|u|^{2} \nu(d u)=1$.

Detailed calculations as those in Example 5.1 reveal that (5.2) has a unique non-explosive weak solution, which is strong Feller continuous and irreducible. Next we verify that $V(x, k):=|x|^{2}+k$ satisfies (4.7) and hence by Proposition 4.6, (5.2) has a unique invariant measure.

Observe that $\nabla V(x, k)=2 x$ and $\nabla^{2} V(x, k)=2 I$. We compute

$$
\begin{aligned}
\mathscr{A} V(x, k):= & \frac{1}{2} \operatorname{tr}\left(a(x, k) \nabla^{2} V(x, k)\right)+\langle b(x, k), \nabla V(x, k)\rangle+\sum_{l \in \mathbb{S}} q_{k l}(x)[V(x, l)-V(x, k)] \\
& +\int_{U}(V(x+c(x, k, u), k)-V(x, k)-\langle\nabla V(x, k), c(x, k, u)\rangle) \nu(d u) \\
\leq & \frac{1}{2} \operatorname{tr}\left(\frac{(|x|+1)^{2}}{16} 2 I\right)-\frac{k}{k+1}\langle x, 2 x\rangle+\sum_{l \neq k} \frac{(2+\cos (k|x|))(l-k)}{3^{l}\left(2+\sin \left(|x|^{2}\right)\right)} \\
& +\frac{k}{k+1} \int_{U} \gamma^{2}|x|^{2}|u|^{2} \nu(d u) \\
= & \frac{(|x|+1)^{2}}{8}-\frac{2 k}{k+1}|x|^{2}+\frac{k}{k+1}|x|^{2}+\frac{2+\cos (k|x|)}{2+\sin \left(|x|^{2}\right)} \sum_{l \neq k} \frac{l-k}{3 l} \\
\leq & \frac{|x|^{2}+1}{4}-\frac{k}{k+1}|x|^{2}+\frac{2+\cos (k|x|)}{2+\sin \left(|x|^{2}\right)}\left(\frac{3}{4}-\frac{k}{2}\right) \\
\leq & -\frac{1}{4}|x|^{2}+\frac{5}{2}-\frac{k}{6} \\
\leq & -\frac{1}{6} V(x, k)+\frac{5}{2},
\end{aligned}
$$

for all $(x, k) \in \mathbb{R}^{2} \times \mathbb{S}$. Since $V(x, k) \rightarrow \infty$ as $|x| \vee k \rightarrow \infty$, this apparently implies (4.7) and hence a unique invariant probability measure $\pi$ for (5.2) exists.

Acknowledgement. The authors would like to thank the Associate Editor and the anonymous reviewers for their helpful comments and suggestions. 


\section{A Proofs of Several Technical Results}

Proof of Lemma 2.1. We will prove the lemma separately for the cases $d=1$ and $d \geq 2$.

Case (i): $d=1$. Let $\left\{a_{n}\right\}$ be a strictly decreasing sequence of real numbers satisfying $a_{0}=1$, $\lim _{n \rightarrow \infty} a_{n}=0$, and $\int_{a_{n}}^{a_{n-1}} \frac{\mathrm{d} r}{r}=n$ for each $n \geq 1$. For each $n \geq 1$, let $\rho_{n}$ be a nonnegative continuous function with support on $\left(a_{n}, a_{n-1}\right)$ so that

$$
\int_{a_{n}}^{a_{n-1}} \rho_{n}(r) \mathrm{d} r=1 \text { and } \rho_{n}(r) \leq 2(n r)^{-1} \text { for all } r>0 .
$$

For $x \in \mathbb{R}$, define

$$
\psi_{n}(x)=\int_{0}^{|x|} \int_{0}^{y} \rho_{n}(z) \mathrm{d} z \mathrm{~d} y .
$$

We can immediately verify that $\psi_{n}$ is even and twice continuously differentiable, with

$$
\psi_{n}^{\prime}(r)=\operatorname{sgn}(r) \int_{0}^{|r|} \rho_{n}(z) \mathrm{d} z=\operatorname{sgn}(r)\left|\psi_{n}^{\prime}(r)\right|,
$$

and

$$
\left|\psi_{n}^{\prime}(r)\right| \leq 1, \quad 0 \leq|r| \psi_{n}^{\prime \prime}(r)=|r| \rho_{n}(|r|) \leq \frac{2}{n}, \quad \text { and } \quad \lim _{n \rightarrow \infty} \psi_{n}(r)=|r|
$$

for $r \in \mathbb{R}$. Furthermore, for each $r>0$, the sequence $\left\{\psi_{n}(r)\right\}_{n \geq 1}$ is nondecreasing. For each $n \in \mathbb{N}$, one can show that $\psi_{n}, \psi_{n}^{\prime}$, and $\psi_{n}^{\prime \prime}$ all vanish on the interval $\left(-a_{n}, a_{n}\right)$. Moreover the classical arguments using Assumption 1.2 (i), (A.2) and (A.3) reveal that

$$
\begin{aligned}
\widetilde{\mathcal{L}}_{k} \psi_{n}(x-z)= & \frac{1}{2} \psi_{n}^{\prime \prime}(x-z)|\sigma(x, k)-\sigma(z, k)|^{2}+\psi_{n}^{\prime}(x-z)(b(x, k)-b(z, k)) \\
& +\int_{U}\left[\psi_{n}(x-z+c(x, k, u)-c(z, k, u))\right. \\
& \left.\quad-\psi_{n}(x-z)-\psi_{n}^{\prime}(x-z)(c(x, k, u)-c(z, k, u))\right] \nu(\mathrm{d} u) \\
\leq & K \frac{\kappa_{R}}{n}+\kappa_{R} \rho(|x-z|),
\end{aligned}
$$

for all $x, z$ with $|x| \vee|z| \leq R$ and $0<|x-z| \leq \delta_{0}$, where $K$ is a positive constant independent of $R$ and $n$. Then it follows that

$$
\begin{aligned}
\mathbb{E}\left[\psi _ { n } \left(\Delta_{\left.\left.t \wedge S_{\delta_{0}} \wedge \tau_{R} \wedge \zeta\right)\right]}\right.\right. & =\mathbb{E}\left[\psi_{n}\left(\tilde{X}\left(t \wedge S_{\delta_{0}} \wedge \tau_{R} \wedge \zeta\right)-X\left(t \wedge S_{\delta_{0}} \wedge \tau_{R} \wedge \zeta\right)\right)\right] \\
& =\psi_{n}(\tilde{x}-x)+\mathbb{E}\left[\int_{0}^{t \wedge \tau_{R} \wedge S_{\delta_{0}} \wedge \zeta} \widetilde{\mathcal{L}}_{k} \psi_{n}(\tilde{X}(s)-X(s)) \mathrm{d} s\right] \\
& \leq \psi_{n}\left(\left|\Delta_{0}\right|\right)+\mathbb{E}\left[\int_{0}^{t \wedge \tau_{R} \wedge S_{\delta_{0}} \wedge \zeta}\left(\kappa_{R} \rho\left(\left|\Delta_{s}\right|\right)+K \frac{\kappa_{R}}{n}\right) \mathrm{d} s\right] \\
& \leq \psi_{n}\left(\left|\Delta_{0}\right|\right)+K \frac{\kappa_{R}}{n} t+\kappa_{R} \int_{0}^{t} \rho\left(\mathbb { E } \left[\mid \Delta_{\left.\left.s \wedge \tau_{R} \wedge S_{\delta_{0}} \wedge \zeta \mid\right]\right) \mathrm{d} s}\right.\right.
\end{aligned}
$$

where the first inequality follows from (A.4) and the second inequality follows from the concavity of $\rho$ and Jensen's inequality. Then we use the monotone convergence theorem and (A.3) to derive

$$
\mathbb{E}\left[\left|\Delta_{t \wedge \tau_{R} \wedge S_{\delta_{0}} \wedge \zeta}\right|\right] \leq\left|\Delta_{0}\right|+\kappa_{R} \int_{0}^{t} \rho\left(\mathbb{E}\left[\left|\Delta_{s \wedge \tau_{R} \wedge S_{\delta_{0}} \wedge \zeta}\right|\right]\right) d s
$$




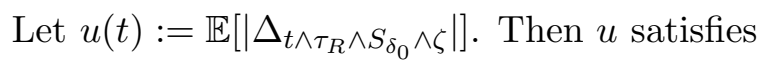

$$
0 \leq u(t) \leq v(t):=\left|\Delta_{0}\right|+\kappa_{R} \int_{0}^{t} \rho(u(s)) d s
$$

Define the function $\Gamma(r):=\int_{1}^{r} \frac{d s}{\rho(s)}$ for $r>0$. Thanks to (1.9), we can verify that $\Gamma$ is nondecreasing and satisfies $\Gamma(r)>-\infty$ for all $r>0$ and $\lim _{r \rightarrow 0} \Gamma(r)=-\infty$. Then we have

$$
\begin{aligned}
\Gamma(u(t)) & \leq \Gamma(v(t))=\Gamma\left(\left|\Delta_{0}\right|\right)+\int_{0}^{t} \Gamma^{\prime}(v(s)) v^{\prime}(s) d s=\Gamma\left(\left|\Delta_{0}\right|\right)+\kappa_{R} \int_{0}^{t} \frac{\rho(u(s))}{\rho(v(s))} d s \\
& \leq \Gamma\left(\left|\Delta_{0}\right|\right)+\kappa_{R} \int_{0}^{t} 1 d s=\Gamma\left(\left|\Delta_{0}\right|\right)+\kappa_{R} t,
\end{aligned}
$$

where we use the assumption that $\rho$ is nondecreasing to obtain the last inequality. Taking the limit $\left|\Delta_{0}\right|=|\tilde{x}-x| \rightarrow 0$ we have $\Gamma(u(t)) \rightarrow-\infty$ since $\lim _{r \rightarrow 0} \Gamma(r)=-\infty$. Moreover, since $\Gamma(r)>-\infty$ for all $r>0$ we must have $\lim _{|\tilde{x}-x| \rightarrow 0} u(t)=0$. This gives (2.8) as desired.

Case (ii) $d \geq 2$. Consider the function $f(x, z):=|x-z|^{2}$. Then Assumption 1.2 (ii) implies that

$$
\begin{aligned}
\widetilde{\mathcal{L}}_{k} f(x, z) & =2\langle x-z, b(x, k)-b(z, k)\rangle+|\sigma(x, k)-\sigma(z, k)|^{2}+\int_{U}|c(x, k, u)-c(z, k, u)|^{2} \nu(d u) \\
& \leq \kappa_{R} \rho\left(|x-z|^{2}\right),
\end{aligned}
$$

for all $x, z \in \mathbb{R}^{d}$ with $|x| \vee|z| \leq R$ and $|x-z| \leq \delta_{0}$. Consequently

$$
\begin{aligned}
\mathbb{E}\left[\mid \Delta_{\left.\left.t \wedge \tau_{R} \wedge S_{\delta_{0}} \wedge \zeta\right|^{2}\right]}\right. & =\mathbb{E}\left[f\left(\tilde{X}\left(t \wedge \tau_{R} \wedge S_{\delta_{0}} \wedge \zeta\right), X\left(t \wedge \tau_{R} \wedge S_{\delta_{0}} \wedge \zeta\right)\right)\right] \\
& =f(\tilde{x}, x)+\mathbb{E}\left[\int_{0}^{t \wedge \tau_{R} \wedge S_{\delta_{0}} \wedge \zeta} \widetilde{\mathcal{L}}_{k} f(\tilde{X}(s), X(s)) d s\right] \\
& \leq\left|\Delta_{0}\right|+\mathbb{E}\left[\int_{0}^{t \wedge \tau_{R} \wedge S_{\delta_{0}} \wedge \zeta} \kappa_{R} \rho\left(|\tilde{X}(s)-X(s)|^{2}\right) d s\right] \\
& \leq\left|\Delta_{0}\right|+\kappa_{R} \int_{0}^{t} \rho\left(\mathbb { E } \left[\mid \Delta_{\left.\left.\left.s \wedge \tau_{R} \wedge S_{\delta_{0}} \wedge \zeta\right|^{2}\right]\right) d s}\right.\right.
\end{aligned}
$$

where the last inequality follows from the concavity of $\rho$ and Jensen's inequality. Using the same argument as that in Case (i), we can show that $\lim _{|\tilde{x}-x| \rightarrow 0} \mathbb{E}\left[\left|\Delta_{t \wedge \tau_{R} \wedge S_{\delta_{0}} \wedge \zeta}\right|^{2}\right]=0$; which, together with Hölder's inequality, leads to (2.8). Combining the two cases completes the proof.

Proof of Lemma 3.2. Let use first prove the lemma for the case when $d \geq 2$. In view of (3.4), it follows from (3.6) that

$$
\begin{aligned}
& \widehat{\Omega}_{\mathrm{d}}^{(k)} G(|x-z|) \\
& \quad=\frac{G^{\prime \prime}(|x-z|)}{2} \bar{A}(x, k, z, k)+\frac{G^{\prime}(|x-z|)}{2|x-z|}[\operatorname{tr} A(x, k, z, k)-\bar{A}(x, k, z, k)+2 B(x, k, z, k)] \\
& \quad \leq \frac{G^{\prime \prime}(|x-z|)}{2} 4 \lambda_{R}+\frac{G^{\prime}(|x-z|)}{2|x-z|}\left[\left|\sigma_{\lambda_{R}}(x, k)-\sigma_{\lambda_{R}}(z, k)\right|^{2}+2 B(x, k, z, k)\right] \\
& \quad=2 \lambda_{R}\left(-1-\frac{\kappa_{R}}{2 \lambda_{R}} g(|x-z|) G^{\prime}(|x-z|)\right)
\end{aligned}
$$




$$
\begin{gathered}
+\frac{G^{\prime}(|x-z|)}{2|x-z|}\left[\left|\sigma_{\lambda_{R}}(x, k)-\sigma_{\lambda_{R}}(z, k)\right|^{2}+2 B(x, k, z, k)\right] \\
=-2 \lambda_{R}+\left(-\kappa_{R} g(|x-z|)+\frac{\left|\sigma_{\lambda_{R}}(x, k)-\sigma_{\lambda_{R}}(z, k)\right|^{2}+2 B(x, k, z, k)}{2|x-z|}\right) G^{\prime}(|x-z|) .
\end{gathered}
$$

Since the function $G$ is concave, we have $G\left(r_{1}\right)-G\left(r_{0}\right) \leq G^{\prime}\left(r_{0}\right)\left(r_{1}-r_{0}\right)$ for all $r_{0}, r_{1} \geq 0$. Take $r_{0}=|x-z|$ and $r_{1}=|x+c(x, k, u)-z-c(z, k, u)|$ to obtain

$$
\begin{aligned}
& G(|x+c(x, k, u)-z-c(z, k, u)|)-G(|x-z|)-\frac{G^{\prime}(|x-z|)}{|x-z|}\langle x-z, c(x, k, u)-c(z, k, u)\rangle \\
& \leq G^{\prime}(|x-z|)\left(|x+c(x, k, u)-z-c(z, k, u)|-|x-z|-\frac{\langle x-z, c(x, k, u)-c(z, k, u)\rangle}{|x-z|}\right) .
\end{aligned}
$$

Furthermore, with $a:=x-z$ and $b:=c(x, k, u)-c(z, k, u)$, we can verify directly that

$$
|a+b|-|a|-\frac{\langle a, b\rangle}{|a|}=\frac{-(|a+b|-|a|)^{2}+|b|^{2}}{2|a|} \leq \frac{|b|^{2}}{2|a|}
$$

Hence it follows that

$$
\begin{aligned}
& G(|x+c(x, k, u)-z-c(z, k, u)|)-G(|x-z|)-\frac{G^{\prime}(|x-z|)}{|x-z|}\langle x-z, c(x, k, u)-c(z, k, u)\rangle \\
& \quad \leq\left(\frac{|c(x, k, u)-c(z, k, u)|^{2}}{2|x-z|}\right) G^{\prime}(|x-z|) .
\end{aligned}
$$

Then we have

$$
\widetilde{\Omega}_{\mathrm{j}}^{(k)} G(|x-z|) \leq G^{\prime}(|x-z|) \int_{U} \frac{|c(x, k, u)-c(z, k, u)|^{2}}{2|x-z|} \nu(d u) .
$$

From (A.5) and (A.6), we see that

$$
\begin{aligned}
\widehat{\mathcal{L}}_{k} G(|x-z|)= & {\left[\widehat{\Omega}_{\mathrm{d}}^{(k)}+\widetilde{\Omega}_{\mathrm{j}}^{(k)}\right] G(|x-z|) } \\
\leq-2 \lambda_{R}+G^{\prime}(|x-z|)( & -\kappa_{R} g(|x-z|)+\frac{\left|\sigma_{\lambda_{R}}(x, i)-\sigma_{\lambda_{R}}(z, j)\right|^{2}+2 B(x, k, z, k)}{2|x-z|} \\
& \left.\quad+\int_{U} \frac{|c(x, k, u)-c(z, k, u)|^{2}}{2|x-z|} \nu(d u)\right) \\
\leq-2 \lambda_{R} . &
\end{aligned}
$$

This gives (3.8) when $d \geq 2$.

Finally we look at the case when $d=1$. First we notice that

$$
\bar{A}(x, i, z, j)=\operatorname{tr} A(x, i, z, j)=\left(\sigma_{\lambda_{R}}(x, i)-\sigma_{\lambda_{R}}(z, j)\right)^{2}+4 \lambda_{R} .
$$

Using this observation in (A.5) gives us

$$
\begin{aligned}
& \widehat{\Omega}_{\mathrm{d}}^{(k)} G(|x-z|) \\
& \quad=\frac{G^{\prime \prime}(|x-z|)}{2} \bar{A}(x, k, z, k)+\frac{G^{\prime}(|x-z|)}{2|x-z|}[\operatorname{tr} A(x, k, z, k)-\bar{A}(x, k, z, k)+2 B(x, k, z, k)] \\
& \quad \leq-2 \lambda_{R}+\left(-\kappa_{R} g(|x-z|)+\frac{2 B(x, k, z, k)}{2|x-z|}\right) G^{\prime}(|x-z|) .
\end{aligned}
$$


The estimation for $\widetilde{\Omega}_{\mathrm{j}}^{(k)} G(|x-z|)$ is the same as before. It then follows from (A.6), (A.7), and (1.19) that

$$
\begin{aligned}
\widehat{\mathcal{L}}_{k} G(|x-z|) \leq & -2 \lambda_{R} \\
& +G^{\prime}(|x-z|)\left(-\kappa_{R} g(|x-z|)+\frac{2 B(x, k, z, k)+\int_{U}|c(x, k, u)-c(z, k, u)|^{2} \nu(d u)}{2|x-z|}\right) \\
\leq & -2 \lambda_{R},
\end{aligned}
$$

again establishing (3.8) for the case when $d=1$. The proof is complete.

Proof of Lemma 4.3. Let $T>0, r>0$ and $x, a \in \mathbb{R}^{d}$ be arbitrary but fixed. We will show that

$$
P^{(k)}(T, x, B(a ; r))=\mathbb{P}\left\{\left|X^{(k)}(T)-a\right|<r \mid X^{(k)}(0)=x\right\}>0
$$

or equivalently $\mathbb{P}\left\{\left|X^{(k)}(T)-a\right| \geq r \mid X^{(k)}(0)=x\right\}<1$. Let us choose some $t_{0} \in(0, T)$. For any $n \in \mathbb{N}$, we set $X_{n}^{(k)}\left(t_{0}\right):=X^{(k)}\left(t_{0}\right) 1_{\left\{\left|X^{(k)}\left(t_{0}\right)\right| \leq n\right\}}$. Since $\lim _{r \rightarrow 0} F(r)=0$ and $0 \leq F \leq 1$, the bounded convergence implies that

$$
\lim _{n \rightarrow \infty} \mathbb{E}\left[F\left(\left|X_{n}^{(k)}\left(t_{0}\right)-X^{(k)}\left(t_{0}\right)\right|^{2}\right)\right]=0 .
$$

For $t \in\left[t_{0}, T\right]$, define

$$
J^{n}(t):=\frac{T-t}{T-t_{0}} X_{n}^{(k)}\left(t_{0}\right)+\frac{t-t_{0}}{T-t_{0}} a, \text { and } h^{n}(t):=\frac{a-X_{n}^{(k)}\left(t_{0}\right)}{T-t_{0}}-b\left(J^{n}(t), k\right) .
$$

Observe that $J^{n}\left(t_{0}\right)=X_{n}^{(k)}\left(t_{0}\right)$ and $J^{n}(T)=a$. In addition, $J^{n}$ satisfies the following stochastic differential equation

$$
J^{n}(t)=X_{n}^{(k)}\left(t_{0}\right)+\int_{t_{0}}^{t} b\left(J^{n}(s), k\right) d s+\int_{t_{0}}^{t} h^{n}(s) d s, \quad t \in\left[t_{0}, T\right] .
$$

Consider the stochastic differential equation

$$
\begin{gathered}
Y(t)=X^{(k)}\left(t_{0}\right)+\int_{t_{0}}^{t}\left[b(Y(s), k)+h^{n}(s)\right] d s+\int_{t_{0}}^{t} \sigma(Y(s), k) d W(s) \\
+\int_{t_{0}}^{t} \int_{U} c(Y(s), k, u) \tilde{N}(d s, d u), \quad t \in\left[t_{0}, T\right] .
\end{gathered}
$$

Also denote $\Delta_{t}:=Y(t)-J^{n}(t)$ for $t \in\left[t_{0}, T\right]$. Note that $\Delta_{t_{0}}=X^{(k)}\left(t_{0}\right)-X_{n}^{(k)}\left(t_{0}\right)$ and $\Delta_{T}=$ $Y(T)-a$. In addition, $\Delta_{t}$ satisfies the stochastic differential equation

$$
\Delta_{t}=\Delta_{t_{0}}+\int_{t_{0}}^{t}\left[b(Y(s), k)-b\left(J^{n}(s), k\right)\right] d s+\int_{t_{0}}^{t} \sigma(Y(s), k) d W(s)+\int_{t_{0}}^{t} \int_{U} c(Y(s), k, u) \tilde{N}(d s, d u) .
$$

Consequently the generator of the process $\Delta_{t}$ is given by

$$
\begin{aligned}
\mathcal{L} f(x) & =\mathcal{L}_{\mathrm{d}} f(x)+\mathcal{L}_{\mathrm{j}} f(x) \\
: & =\frac{1}{2} \operatorname{tr}\left(\sigma(Y(s), k) \sigma(Y(s), k)^{T} \nabla^{2} f(x)\right)+\left\langle b(Y(s), k)-b\left(J^{n}(s), k\right), \nabla f(x)\right\rangle
\end{aligned}
$$




$$
+\int_{U}(f(x+c(Y(s), k, u))-f(x)-\langle\nabla f(x), c(Y(s), k, u)\rangle) \nu(d u), \quad f \in C_{c}^{2}\left(\mathbb{R}^{d}\right)
$$

We compute

$$
\begin{aligned}
\mathcal{L}_{\mathrm{d}} F\left(\left|\Delta_{s}\right|^{2}\right)= & \frac{1}{2} \operatorname{tr}\left(\sigma(Y(s), k) \sigma(Y(s), k)^{T} \nabla^{2} F\left(\left|\Delta_{s}\right|^{2}\right)\right)+\left\langle b(Y(s), k)-b\left(J^{n}(s), k\right), \nabla F\left(\left|\Delta_{s}\right|^{2}\right)\right\rangle \\
= & \frac{1}{2} \operatorname{tr}\left(\sigma(Y(s), k) \sigma(Y(s), k)^{T}\left[4 F^{\prime \prime}\left(\left|\Delta_{s}\right|^{2}\right) \Delta_{s} \Delta_{s}^{T}+2 F^{\prime}\left(\left|\Delta_{s}\right|^{2}\right) I\right]\right) \\
& +\left\langle b(Y(s), k)-b\left(J^{n}(s), k\right), 2 F^{\prime}\left(\left|\Delta_{s}\right|\right) \Delta_{s}\right\rangle \\
= & 2 F^{\prime \prime}\left(\left|\Delta_{s}\right|^{2}\right)\left|\Delta_{s}^{T} \sigma(Y(s), k)\right|^{2}+F^{\prime}\left(\left|\Delta_{s}\right|\right)|\sigma(Y(s), k)|^{2} \\
& +2 F^{\prime}\left(\left|\Delta_{s}\right|^{2}\right)\left\langle b(Y(s), k)-b\left(J^{n}(s), k\right), \Delta_{s}\right\rangle \\
\leq & F^{\prime}\left(\left|\Delta_{s}\right|\right)\left[|\sigma(Y(s), k)|^{2}+2\left\langle b(Y(s), k)-b\left(J^{n}(s), k\right), \Delta_{s}\right\rangle\right]
\end{aligned}
$$

where the inequality follows from (4.5). Likewise, the concavity of $F$ leads to

$$
\begin{aligned}
\mathcal{L}_{\mathrm{j}} F\left(\left|\Delta_{s}\right|^{2}\right) & =\int_{U}\left(F\left(\left|\Delta_{s}+c(Y(s), k, u)\right|^{2}\right)-F\left(\left|\Delta_{s}\right|^{2}\right)-\left\langle\nabla F\left(\left|\Delta_{s}\right|^{2}\right), c(Y(s), k, u)\right\rangle\right) \nu(d u) \\
& \leq \int_{U}\left[F^{\prime}\left(\left|\Delta_{s}\right|^{2}\right)\left[\left|\Delta_{s}+c(Y(s), k, u)\right|^{2}-\left|\Delta_{s}\right|^{2}\right]-2 F^{\prime}\left(\left|\Delta_{s}\right|^{2}\right)\left\langle\Delta_{s}, c(Y(s), k, u)\right\rangle\right] \nu(d u) \\
& =\int_{U} F^{\prime}\left(\left|\Delta_{s}\right|^{2}\right)|c(Y(s), k, u)|^{2} \nu(d u) .
\end{aligned}
$$

Therefore, by adding the above two inequalities, we have

$$
\mathcal{L} F\left(\left|\Delta_{s}\right|^{2}\right) \leq F^{\prime}\left(\left|\Delta_{s}\right|^{2}\right)\left[|\sigma(Y(s), k)|^{2}+2\left\langle b(Y(s), k)-b\left(J^{n}(s), k\right), \Delta_{s}\right\rangle+\int_{U}|c(Y(s), k, u)|^{2} \nu(d u)\right] .
$$

On the other hand, when $|Y(s)| \leq R,\left|J^{n}(s)\right| \leq R$ and $\left|\Delta_{s}\right| \leq \delta_{0}$, we can use (1.20) and (1.22) to obtain

$$
\begin{aligned}
|\sigma(Y(s), k)|^{2}+2\langle b(Y(s), k) & \left.-b\left(J^{n}(s), k\right), \Delta_{s}\right\rangle+\int_{U}|c(Y(s), k, u)|^{2} \nu(d u) \\
& \leq \kappa\left(|Y(s)|^{2}+1\right)+2 \kappa_{R}\left|\Delta_{s}\right| g\left(\left|\Delta_{s}\right|\right) \leq K_{R}+\kappa|Y(s)|^{2}
\end{aligned}
$$

where $K_{R}=\kappa+2 \kappa_{R} \max _{r \in\left[0, \delta_{0}\right]}\{r g(r)\}<\infty$. Then it follows that

$$
\mathcal{L} F\left(\left|\Delta_{s}\right|^{2}\right) \leq F^{\prime}\left(\left|\Delta_{s}\right|^{2}\right)\left[K_{R}+\kappa|Y(s)|^{2}\right] \leq K_{R}+\kappa|Y(s)|^{2} .
$$

In view of (1.22) and (4.1), we can use the standard arguments to show that $\mathbb{E}\left[\sup _{t_{0} \leq s \leq T}|Y(s)|^{2}\right] \leq$ $K$, where $K$ is a positive constant independent of $t_{0}$. For any $R>0$, we define $\tau_{R}:=\inf \left\{t \geq t_{0}\right.$ : $\left.|Y(t)| \vee\left|J^{n}(t)\right|>R\right\} \wedge T$ and $S_{\delta_{0}}:=\inf \left\{t \geq t_{0}:\left|Y(t)-J^{n}(t)\right| \geq \delta_{0}\right\} \wedge T$. Then we can compute

$$
\begin{aligned}
\mathbb{E}\left[F\left(\left|\Delta_{T \wedge \tau_{R} \wedge S_{\delta_{0}}}\right|^{2}\right)\right] & =\mathbb{E}\left[F\left(\left|\Delta_{t_{0}}\right|^{2}\right)\right]+\mathbb{E}\left[\int_{t_{0}}^{T \wedge \tau_{R} \wedge S_{\delta_{0}}} \mathcal{L} F\left(\left|\Delta_{s^{-}}\right|^{2}\right) d s\right] \\
& \leq \mathbb{E}\left[F\left(\left|\Delta_{t_{0}}\right|^{2}\right)\right]+\mathbb{E}\left[\int_{t_{0}}^{T \wedge \tau_{R} \wedge S_{\delta_{0}}}\left(K_{R}+\kappa\left|Y\left(s^{-}\right)\right|^{2}\right) d s\right] \\
& \leq \mathbb{E}\left[F\left(\left|\Delta_{t_{0}}\right|^{2}\right)\right]+K_{R}\left(T-t_{0}\right)+\mathbb{E}\left[\int_{t_{0}}^{T} \kappa|Y(s)|^{2} d s\right]
\end{aligned}
$$




$$
\leq \mathbb{E}\left[F\left(\left|\Delta_{t_{0}}\right|^{2}\right)\right]+\left(K_{R}+\kappa K\right)\left(T-t_{0}\right)
$$

Next we show that

$$
\mathbb{E}\left[F\left(\left|\Delta_{T}\right|^{2}\right)\right] \leq \frac{1}{F\left(\delta_{0}^{2}\right)} \mathbb{E}\left[F\left(\left|\Delta_{T \wedge S_{\delta_{0}}}\right|^{2}\right)\right]
$$

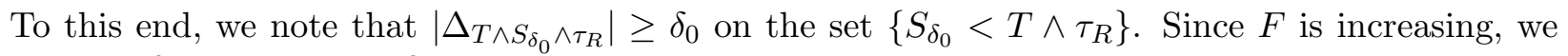
have $F\left(\delta_{0}^{2}\right) \leq F\left(\left|\Delta_{T \wedge S_{\delta_{0}}}\right|^{2}\right)$. This together with the fact that $0 \leq F \leq 1$ give the following

$$
\begin{aligned}
& \frac{\mathbb{E}\left[F\left(\left|\Delta_{T \wedge \tau_{R} \wedge S_{\delta_{0}}}\right|^{2}\right)\right]}{F\left(\delta_{0}^{2}\right)}-\mathbb{E}\left[F\left(\left|\Delta_{T \wedge \tau_{R}}\right|^{2}\right)\right] \\
& \quad=\frac{\mathbb{E}\left[F\left(\left|\Delta_{T \wedge \tau_{R} \wedge S_{\delta_{0}}}\right|^{2}\right) 1_{\left\{T \wedge \tau_{R} \leq S_{\delta_{0}}\right\}}\right]+\mathbb{E}\left[F\left(\left|\Delta_{T \wedge \tau_{R} \wedge S_{\delta_{0}}}\right|^{2}\right) 1_{\left\{T \wedge \tau_{R}>S_{\delta_{0}}\right\}}\right]}{F\left(\delta_{0}^{2}\right)}-\mathbb{E}\left[F\left(\left|\Delta_{T \wedge \tau_{R}}\right|^{2}\right)\right] \\
& \quad \geq \frac{\mathbb{E}\left[F\left(\left|\Delta_{T \wedge \tau_{R}}\right|^{2}\right) 1_{\left\{T \wedge \tau_{R} \leq S_{\delta_{0}}\right\}}\right]+F\left(\delta_{0}^{2}\right) \mathbb{P}\left\{T \wedge \tau_{R}>S_{\delta_{0}}\right\}}{F\left(\delta_{0}^{2}\right)}-\mathbb{E}\left[F\left(\left|\Delta_{T \wedge \tau_{R}}\right|^{2}\right)\right] \\
& \quad \geq \mathbb{P}\left\{T \wedge \tau_{R}>S_{\delta_{0}}\right\}+\mathbb{E}\left[F\left(\left|\Delta_{T \wedge \tau_{R}}\right|^{2}\right) 1_{\left\{T \wedge \tau_{R} \leq S_{\delta_{0}}\right\}}\right]-\mathbb{E}\left[F\left(\left|\Delta_{T \wedge \tau_{R}}\right|^{2}\right)\right] \\
& \quad=\mathbb{P}\left\{T \wedge \tau_{R}>S_{\delta_{0}}\right\}-\mathbb{E}\left[F\left(\left|\Delta_{T \wedge \tau_{R}}\right|^{2}\right) 1_{\left\{T \wedge \tau_{R}>S_{\delta_{0}}\right\}}\right] \\
& \quad \geq \mathbb{P}\left\{T \wedge \tau_{R}>S_{\delta_{0}}\right\}-\mathbb{E}\left[1 \cdot 1_{\left\{T \wedge \tau_{R}>S_{\delta_{0}}\right\}}\right]=0 .
\end{aligned}
$$

Consequently we have $\mathbb{E}\left[F\left(\left|\Delta_{T \wedge \tau_{R}}\right|^{2}\right)\right] \leq \frac{\mathbb{E}\left[F\left(\left|\Delta_{T \wedge \tau_{R} \wedge S_{\delta_{0}}}\right|^{2}\right)\right]}{F\left(\delta_{0}^{2}\right)}$. Since $\lim _{R \rightarrow \infty} \tau_{R}=T$ a.s. and $0 \leq F \leq$ 1, the bounded convergence theorem gives (A.11).

Recall that $Y$ satisfies the stochastic differential equation (A.9) for $t \in\left[t_{0}, T\right]$. For $t \in\left[0, t_{0}\right]$, we define $Y(t):=X^{(k)}(t)$ and $X^{(k)}(t)$ is the weak solution to (1.21) with initial condition $x$. Then the process $Y$ satisfies the following stochastic differential equation:

$$
Y(t)=x+\int_{0}^{t}\left[b(Y(s), k)+h^{n}(s) 1_{\left\{s>t_{0}\right\}}\right] d s+\int_{0}^{t} \sigma(Y(s), k) d W(s)+\int_{0}^{t} \int_{U} c(Y(s), k, u) \tilde{N}(d s, d u)
$$

for $t \in[0, T]$. Next we set

$$
H(t):=1_{\left\{t>t_{0}\right\}} \sigma^{-1}(Y(t), k) h^{n}(t), \text { and } M(t):=\exp \left\{\int_{0}^{t}\langle H(s), d W(s)\rangle-\frac{1}{2} \int_{0}^{t}|H(s)|^{2} d s\right\} .
$$

As argued in Qiao (2014), it follows from (1.23) that $|H(t)|^{2}$ is bounded and hence $M$ is a martingale under $\mathbb{P}$ by Novikov's criteria. Moreover, $\mathbb{E}[M(T)]=1$. Define

$$
\begin{aligned}
\mathbb{Q}(B) & :=\mathbb{E}\left[M(T) 1_{\{B\}}\right], \quad B \in \mathcal{F}_{T} \\
\tilde{W}(t) & :=W(t)+\int_{0}^{t} H(s) d s .
\end{aligned}
$$

It follows from Theorem 132 of Situ $(2005)$ that $\mathbb{Q}$ is a probability measure, $\tilde{W}$ is a $\mathbb{Q}$-Brownian motion and $\tilde{N}(d t, d u)$ is a $\mathbb{Q}$-compensated Poisson random measure with compensator $d t \nu(d u)$. Furthermore, under the measure $\mathbb{Q}, Y$ solves the following stochastic differential equation

$$
Y(t)=x+\int_{0}^{t} b(Y(s), k) d s+\int_{0}^{t} \sigma(Y(s), k) d \tilde{W}(s)+\int_{0}^{t} \int_{U} c(Y(s), k, u) \tilde{N}(d s, d u)
$$


for $t \in[0, T]$. By the uniqueness in law of the solution to the SDE, we have that the law of $\left\{X^{(k)}(t): t \in[0, T]\right\}$ under $\mathbb{P}$ is the same as the law of $\{Y(t): t \in[0, T]\}$ under $\mathbb{Q}$. In particular, we have $\mathbb{P}\left\{\left|X^{(k)}(T)-a\right| \geq r \mid X^{(k)}(0)=x\right\}=\mathbb{Q}\{|Y(T)-a| \geq r \mid Y(0)=x\}$. Since $\mathbb{P}$ and $\mathbb{Q}$ are equivalent, the desired assertion $\mathbb{P}\left\{\left|X^{(k)}(T)-a\right| \geq r \mid X^{(k)}(0)=x\right\}=\mathbb{Q}\{|Y(T)-a| \geq r \mid Y(0)=x\}<1$ will follow if we can show that $\mathbb{P}\{|Y(T)-a| \geq r \mid Y(0)=x\}<1$. To this end, for any $\varepsilon>0$, we first choose an $R>0$ sufficiently large so that $\mathbb{P}\left\{\tau_{R}<T\right\}<\varepsilon$. Sine $F$ is bounded and increasing, we can use (A.11) and (A.10) to compute

$$
\begin{aligned}
\mathbb{P}\{|Y(T)-a| \geq r \mid Y(0)=x\} & =\mathbb{P}\left\{|Y(T)-a|^{2} \geq r^{2} \mid Y(0)=x\right\} \\
& =\mathbb{P}\left\{F\left(|Y(T)-a|^{2}\right) \geq F\left(r^{2}\right) \mid Y(0)=x\right\} \\
& \leq \frac{\mathbb{E}\left[F\left(|Y(T)-a|^{2}\right)\right]}{F\left(r^{2}\right)} \\
& =\frac{\mathbb{E}\left[F\left(\left|\Delta_{T}\right|^{2}\right)\right]}{F\left(r^{2}\right)} \\
& \leq \frac{\mathbb{E}\left[F\left(\left|\Delta_{T \wedge S_{\delta_{0}}}\right|^{2}\right)\right]}{F\left(r^{2}\right) F\left(\delta_{0}^{2}\right)} \\
& =\frac{\mathbb{E}\left[F\left(\left|\Delta_{T \wedge S_{\delta_{0}} \wedge \tau_{R}}\right|^{2}\right) 1_{\left\{\tau_{R} \geq T \wedge S_{\delta_{0}}\right\}}\right]+\mathbb{E}\left[F\left(\left|\Delta_{T \wedge S_{\delta_{0}}}\right|^{2}\right) 1_{\left\{\tau_{R}<T \wedge S_{\delta_{0}}\right\}}\right]}{F\left(r^{2}\right) F\left(\delta_{0}^{2}\right)} \\
& \leq \frac{\mathbb{E}\left[F\left(\left|\Delta_{t_{0}}\right|^{2}\right)\right]+\left(K_{R}+\kappa K\right)\left(T-t_{0}\right)+\mathbb{P}\left\{\tau_{R}<T\right\}}{F\left(r^{2}\right) F\left(\delta_{0}^{2}\right)} \\
& \leq \frac{\mathbb{E}\left[F\left(\left|\Delta_{t_{0}}\right|^{2}\right)\right]+\left(K_{R}+\kappa K\right)\left(T-t_{0}\right)+\varepsilon}{F\left(r^{2}\right) F\left(\delta_{0}^{2}\right)} .
\end{aligned}
$$

Thanks to (A.8), we have $\mathbb{E}\left[F\left(\left|\Delta_{t_{0}}\right|^{2}\right)\right] \rightarrow 0$ as $n \rightarrow \infty$. Therefore we can choose $n$ sufficiently large and $t_{0}$ close enough to $T$ to make the last term less than 1 as desired.

\section{References}

Cerrai, S. (2001). Second order PDE's in finite and infinite dimension: A probabilistic approach, volume 1762 of Lecture Notes in Mathematics. Springer-Verlag, Berlin.

Chen, M. F. \& Li, S. F. (1989). Coupling methods for multidimensional diffusion processes. Ann. Probab., 17(1), 151-177.

Dynkin, E. (1965). Markov Processes, volume 121 of Die Grundlehren der Mathematischen Wissenschaften. Berlin: Springer-Verlag.

Foguel, S. R. (1969). Positive operators on C(X). Proc. Amer. Math. Soc., 22, 295-297.

Hairer, M. (2016). Convergence of markov processes. http://www.hairer.org/notes/Convergence.pdf. Lecture notes, Univ. Warwick.

Mao, X. \& Yuan, C. (2006). Stochastic differential equations with Markovian switching. London: Imperial College Press.

Meyn, S. P. \& Tweedie, R. L. (1992). Stability of Markovian processes. I. Criteria for discrete-time chains. Adv. in Appl. Probab., $24(3), 542-574$. 
Meyn, S. P. \& Tweedie, R. L. (1993a). Stability of Markovian processes. II. Continuous-time processes and sampled chains. Adv. in Appl. Probab., 25(3), 487-517.

Meyn, S. P. \& Tweedie, R. L. (1993b). Stability of Markovian processes. III. Foster-Lyapunov criteria for continuous-time processes. Adv. in Appl. Probab., 25(3), 518-548.

Nguyen, D. H., Yin, G., \& Zhu, C. (2017). Certain properties related to well posedness of switching diffusions. Stochastic Process. Appl., 127(3135-3158).

Peszat, S. \& Zabczyk, J. (1995). Strong Feller property and irreducibility for diffusions on Hilbert spaces. Ann. Probab., 23(1), 157-172.

Priola, E. \& Wang, F.-Y. (2006). Gradient estimates for diffusion semigroups with singular coefficients. J. Funct. Anal., 236(1), 244-264.

Qiao, H. (2014). Exponential ergodicity for SDEs with jumps and non-Lipschitz coefficients. $J$. Theoret. Probab., 27(1), 137-152.

Shao, J. (2015a). Ergodicity of regime-switching diffusions in Wasserstein distances. Stochastic Process. Appl., 125(2), 739-758.

Shao, J. (2015b). Strong solutions and strong Feller properties for regime-switching diffusion processes in an infinite state space. SIAM J. Control Optim., 53(4), 2462-2479.

Shao, J. \& Xi, F. (2014). Stability and recurrence of regime-switching diffusion processes. SIAM J. Control Optim., 52(6), 3496-3516.

Situ, R. (2005). Theory of stochastic differential equations with jumps and applications. Mathematical and Analytical Techniques with Applications to Engineering. Springer, New York.

Stettner, L. (1986). On the existence and uniqueness of invariant measure for continuous time markov processes. Technical Report LCDS \#86-18, Brown University, Providence, RI.

Tuong, T. D., Nguyen, D. H., Dieu, N. T., \& Tran, K. (2019). Extinction and permanence in a stochastic SIRS model in regime-switching with general incidence rate. Nonlinear Anal. Hybrid Syst., 34, 121-130.

Xi, F. (2004). Stability of a random diffusion with nonlinear drift. Statist. Probab. Lett., 68(3), $273-286$.

Xi, F., Yin, G., \& Zhu, C. (2019). Regime-switching jump diffusions with non-Lipschitz coefficients and countably many switching states: existence and uniqueness, Feller, and strong Feller properties. In Modeling, stochastic control, optimization, and applications, volume 164 of IMA Vol. Math. Appl. (pp. 571-599). Springer, Cham.

Xi, F. \& Zhu, C. (2017). On Feller and strong Feller properties and exponential ergodicity of regime-switching jump diffusion processes with countable regimes. SIAM J. Control Optim., 55(3), 1789-1818.

Xi, F. \& Zhu, C. (2019). Jump type stochastic differential equations with non-Lipschitz coefficients: non-confluence, Feller and strong Feller properties, and exponential ergodicity. J. Differential Equations, 266(8), 4668-4711.

Yin, G. G. \& Zhu, C. (2010). Hybrid Switching Diffusions: Properties and Applications, volume 63 of Stochastic Modelling and Applied Probability. New York: Springer.

Zhang, Q. (2001). Stock trading: an optimal selling rule. SIAM J. Control Optim., 40(1), 64-87. 